\title{
Radial asymmetries around the visual field: From retina to cortex to behavior
}

\author{
Authors \\ Eline R. Kupers ${ }^{1 *}$, Noah C. Benson ${ }^{1,2,3}$, Marisa Carrasco ${ }^{1,2}$, Jonathan Winawer ${ }^{1,2}$
}

\author{
Affiliations \\ ${ }^{1}$ Dept. of Psychology, New York University, New York, NY 10003 \\ ${ }^{2}$ Center for Neural Sciences, New York University, New York, NY 10003 \\ ${ }^{3}$ Current affiliation: eScience Institute, University of Washington, Seattle, WA, 98122 \\ * Corresponding author. Email to: eline.kupers@nyu.edu
}

\section{Funding}

This work was supported by NIH NEI RO1-EY027401 to MC and JW.

\section{Acknowledgements}

We thank Michael Landy and Brian Wandell for their useful comments. 


\section{Abstract}

Visual performance varies around the visual field. It is best near the fovea compared to the periphery, and at iso-eccentric locations it is best on the horizontal, intermediate on the lower, and poorest on the upper meridian. The fovea-to-periphery performance decline is linked to the decreases in cone density, retinal ganglion cell (RGC) density, and V1 cortical magnification factor (CMF) as eccentricity increases. The origins of radial asymmetries are not well understood. Optical quality and cone density vary across the retina, but recent computational modeling has shown that these factors can only account for a small percentage of behavior. Here, we investigate how visual processing beyond the cones contributes to radial asymmetries in performance. First, we quantify the extent of asymmetries in cone density, midget RGC density, and V1-V2 CMF. We find that both radial asymmetries and eccentricity gradients are amplified from cones to mRGCs, and from mRGCs to cortex. Second, we extend our previously published computational observer model to quantify the contribution of spatial filtering by mRGCs to behavioral asymmetries. Starting with photons emitted by a visual display, the model simulates the effect of human optics, fixational eye movements, cone isomerizations and mRGC spatial filtering. The model performs a forced choice orientation discrimination task on $\mathrm{mRGC}$ responses using a linear support vector machine classifier. The model shows radial asymmetries in performance that are larger than those from a model operating on the cone outputs, but considerably smaller than those observed from human performance. We conclude that spatial filtering properties of mRGCs contribute to radial performance differences, but that a full account of these differences will entail a large contribution from cortical representations. 


\section{Introduction}

Visual performance is not uniform across the visual field. The most well-known effect is a decrease in visual acuity as a function of eccentricity: we see more poorly in the periphery compared to the center of gaze [1-4]. This observed difference in visual performance has been attributed to several physiological factors, starting as early as the distribution of photoreceptors $[5,6]$. In the human fovea, the cones are tightly packed such that visual input is encoded at high spatial resolution. In peripheral retinal locations, cones are larger and interspersed among rods, resulting in a drastically lower density $[7,8]$; hence a decrease in spatial resolution.

Visual performance also differs as a function of polar angle. At iso-eccentric locations, performance is better along the horizontal than vertical meridian (horizontal-vertical anisotropy or "HVA" e.g., [9-12]) and better along the lower than upper vertical meridian (vertical-meridian asymmetry or "VMA" [10-14]). These radial asymmetries are observed in many different visual tasks, such as those mediated by contrast sensitivity [10-12, 15-28] and spatial resolution [9, 13, $15,16,29-32$ ], contrast appearance [33], visual search [34-42], crowding [14, 42-45], and tasks that are thought to recruit higher visual areas such as visual working memory [31].

These polar angle effects can be large. For instance, for a Gabor patch at $4.5^{\circ}$ eccentricity with a spatial frequency of 4 cycles per degree, contrast thresholds are close to double for the upper vertical meridian compared to the horizontal meridian $[10,11,28]$. This is an effect size similar to doubling stimulus' eccentricity from $4.5^{\circ}$ to $9^{\circ}$ on the horizontal axis [16, 28].

The visual system has radial asymmetries from its earliest stages, including optics and cone density. However, with a computational observer model, we recently showed that variations in optical quality and cone density likely accounted for less than $\sim 10 \%$ of the observed radial asymmetries in a contrast threshold task [46]. This leads to the question, what additional factors later in the visual processing stream give rise to visual performance differences with polar angle?

In the human retina, the best described retinal ganglion cells (RGCs) are the midget and parasol cells. Both of these cell types show a decrease in density as a function of eccentricity and vary in density as a function polar angle in humans [47-53] and monkeys [54-57]. Because midget RGCs are the most numerous ganglion cells in primates (i.e. $80 \%$ of $\sim 1$ million RGCs compared to $10 \%$ parasols and $10 \%$ other types) and have small cell bodies and small dendritic field trees that increase with eccentricity $[55,56,58]$, they are often hypothesized to set an 
anatomical limit on high resolution spatial vision such as acuity and contrast sensitivity at mid to high spatial frequencies $[50,56]$.

Interestingly, in the range of eccentricities used for many psychophysical tasks $\left(0-10^{\circ}\right)$, cone density shows an HVA (greater density on the horizontal than vertical meridian), but not a VMA, inconsistent with behavior (there is a slightly greater density on the upper than lower vertical meridian, opposite what one would predict to explain behavior). Midget RGC density, in contrast, shows both an HVA and a VMA, making their distribution patterns more similar to behavioral patterns.

Here, we investigate to what extent visual performance differences can be explained by the radial variations observed in $\mathrm{mRGC}$ visual field sampling. First, we quantify asymmetries in spatial sampling around the visual field in three early visual processing stages: cones, mRGCs, and V1-V2 cortex. We do so because it is important to first identify if there are any differences in spatial encoding across these processing stages, and if so, how these differences relate to differences in behavior. Then we extend our previously published computational observer model, which included optics, cone sampling, and eye movements, by adding mRGC-like spatial filtering. We compare this observer model to our previous model (no RGC layer) and to human performance on a 2-AFC orientation discrimination task. By comparing the predicted performance to human observers, we can quantify the contribution of $m R G C s$ to visual performance differences around the visual field. 


\section{Results}

We quantify the asymmetries in cone density, midget retinal ganglion cells (mRGCs) density and V1-V2 cortical magnification factor (CMF) - both as a function of eccentricity and for the four cardinal meridians. In the next two sections, we first show that both eccentricity gradients and radial asymmetries are amplified from cones to mRGCs and from mRGCs to early visual cortex. Then we implement the observed variations in mRGC density in a computational observer model to test whether biologically plausible differences in mRGC sampling across the cardinal meridians can quantitatively explain psychophysical performance differences as a function of polar angle.

\section{Fovea-to-periphery gradient is amplified from retina to mRGCs to early visual cortex}

A hallmark of the human retina is the sharp drop in cone density from fovea to periphery $[7,8$, 59]. Within the central one degree, cone density decreases dramatically (on average by 3.5 fold). Beyond the fovea, cone density continues to decrease by 10 -fold between $1^{\circ}$ and $20^{\circ}$ eccentricity (Fig 1A, left panel). This decrease in cone density is due to an increase in cone spacing caused by the presence of rods and by the increase in cone diameter [7].

The second processing stage we focus on are the midget RGCs. The mRGC cell bodies are laterally displaced from their receptive fields by the foveal cones. Therefore, we use a computational model by Watson [60] that combines cone density [7], mRGC density [48] and displacement [52] to infer the mRGC density referred to the visual field, rather than the cell body positions. Throughout, when we refer to $\mathrm{mRGC}$ density, it is always with respect to receptive fields. Like the cones, midget RGCs sample the visual field differentially as a function of eccentricity. The fovea-to-periphery gradient is steeper for mRGCs than for cones (Fig 1A, middle panel). At the central one degree, mRGC density is greater than cone density. This divergence results in a cone:mRGC ratio of 0.5 (Fig 1B, left panel), indicating a 'direct line' between a single cone and a pair of on- and off-center mRGCs. In the periphery, mRGC density falls off at a faster rate than cones. For example, cone density decreases by 10 -fold between $1^{\circ}$ and $20^{\circ}$ eccentricity, whereas $\mathrm{mRGC}$ density decreases by 80 -fold. This convergence can also be expressed in the cone:mRGC ratio, which increases as a function of eccentricity (Fig 1B, left panel). 

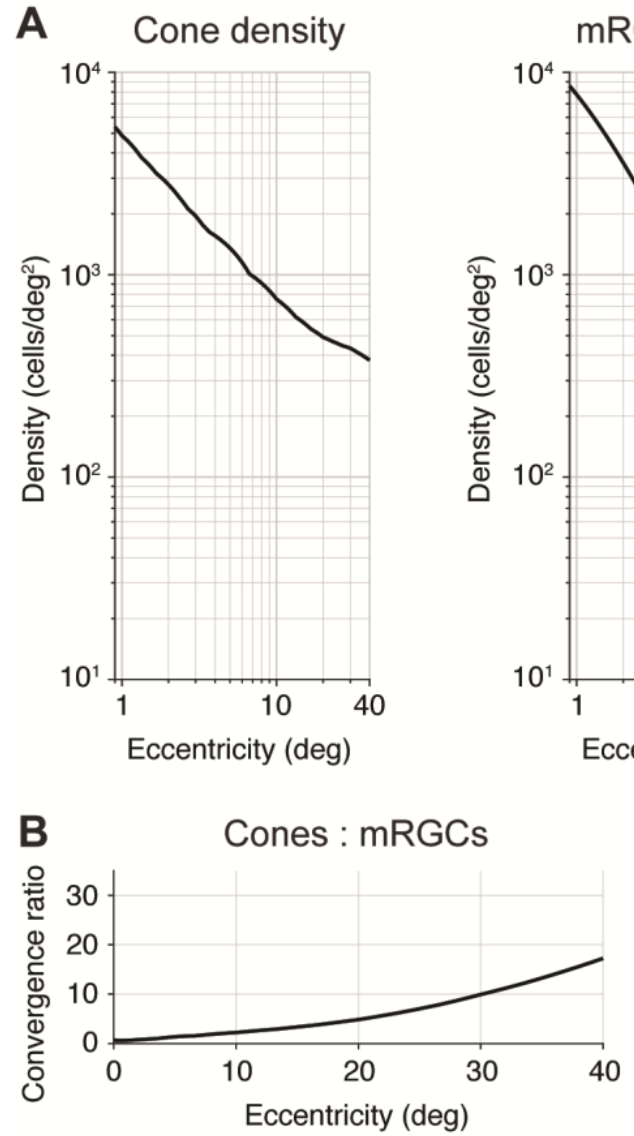

mRGC density

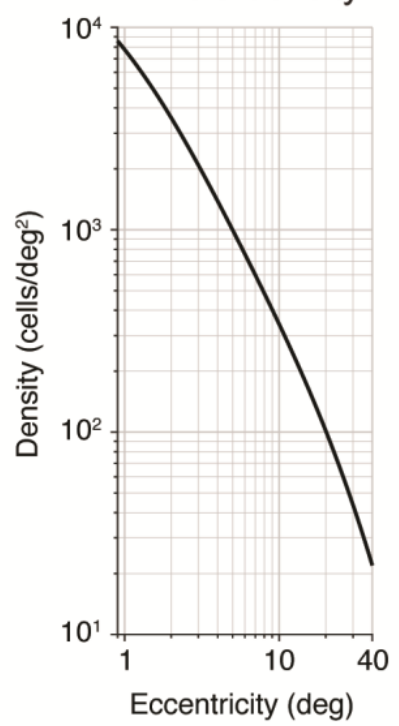

V1 CMF

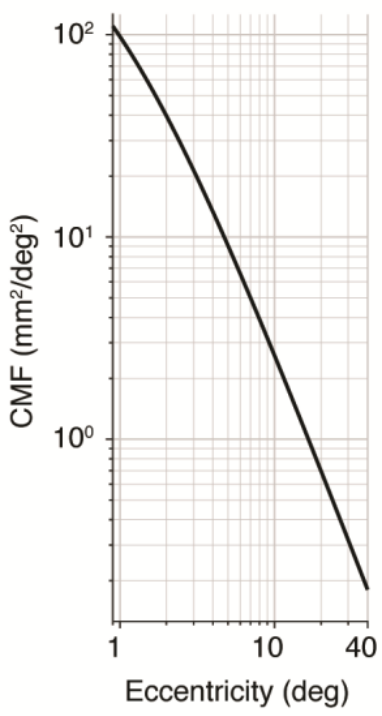

mRGCs : V1 CMF

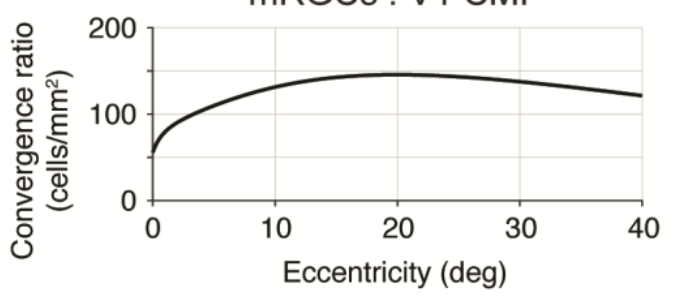

Fig 1. Foveal over-representation is amplified from cones to mRGCs to cortex. (A) Cone density, mRGC receptive field density and V1 cortical magnification factor as a function of eccentricity. Left panel: Cone data from Curcio et al. [7]. Middle panel: midget RGC RF density data from Watson [60]. Both cone and mRGC data are the average across cardinal retinal meridians of the left eye using the publicly available toolbox ISETBIO. Right panel: V1 CMF is predicted by the areal equation published in Horton \& Hoyt [61]. (B) Transformation ratios from cones to mRGCs and mRGCs to V1. The cone:mRGC ratio is unitless, as both cone density and mRGC density are quantified in cells/deg ${ }^{2}$. The increasing ratio indicates higher convergence of cone signals by the mRGCs. For mRGC:V1 CMF ratio units are defined in cells $/ \mathrm{mm}^{2}$. The ratio increase in the first 20 degrees indicates an amplification of the foveal over-representation in V1 compared to mRGCs.

Third, we quantify the amount of V1 surface area devoted to a portion of the visual field, also known as the cortical magnification factor (Fig 1A, right panel). There have been claims that V1 CMF is proportional to retinal ganglion cell density, as first proposed by Polyak [62, 63] (for an overview, see [64, 65] and see Discussion). However, when comparing mRGCs density [60] to human V1 CMF [61], we find that the ratio is not constant: The foveal magnification is even more accentuated in V1 (Fig 1B, right panel). These results are consistent with the findings in squirrel monkey [66]; owl monkey [67], and macaque [68], all of which show that the cortical magnification function falls off with eccentricity more steeply in V1 than would be predicted by mRGC density alone. 


\section{Radial asymmetries are amplified from cones to mRGCs}

Cone density differs as a function of polar angle. It is higher along the nasal and temporal retina meridians (representing the horizontal visual meridian) than the inferior and superior retina meridians (representing the upper and lower vertical visual meridian) (Fig 2A, left panel). This horizontal-vertical asymmetry is around $20 \%$ and relatively constant with eccentricity. There is no systematic difference between the cone density in the inferior and superior retina. If anything, there is a slight 'inverted' vertical-meridian asymmetry in the central five degrees: cones are more densely packed along the upper vertical meridian (inferior retina). Assuming greater density leads to better performance, this would predict better performance on the upper vertical meridian in the central three degrees, opposite of the typical asymmetry reported in behavior, which has been found up to $1.5^{\circ}$ eccentricity in a study on contrast sensitivity [26]. All of these patterns of cone density asymmetries are found using two different datasets with different methods: a post-mortem retinal dataset by Curcio et al. [7] and an in vivo dataset from Song et al. [8], indicating reproducibility of the biological finding. All of the patterns are also consistent when computed using two different analysis toolboxes (ISETBIO [69-71] and rgcDisplacementMap [72], Supplemental Fig 1, top row), indicating computational reproducibility.

The radial asymmetries in cone density are larger in the $\mathrm{mRGC}$ distribution. The nasal and temporal retina contain higher cell densities (after correction for cell body displacement) compared to inferior and superior retina (Fig 2A, middle panel). This horizontal-vertical asymmetry increases with eccentricity. For example, at $3.5^{\circ}$ eccentricity, the average nasal and temporal density is $\sim 20 \%$ higher compared to the average of inferior and superior retina. By $40^{\circ}$ eccentricity, this density difference increases to $\sim 60 \%$. Beyond $10^{\circ}$ eccentricity, this horizontalvertical asymmetry is mostly driven by the nasal retina, as it contains higher mRGC density compared to the temporal retina. This finding is in line with earlier histology reports in macaque [57] and positively correlated with spatial resolution tasks (e.g. [73]). This nasal-temporal asymmetry, although interesting, is beyond the focus of this paper, as the asymmetries in performance we observe are found in both binocular and monocular experiments [10, 32]. Overall, the greater emphasis on the horizontal is substantially greater in the mRGCs than the cones. 

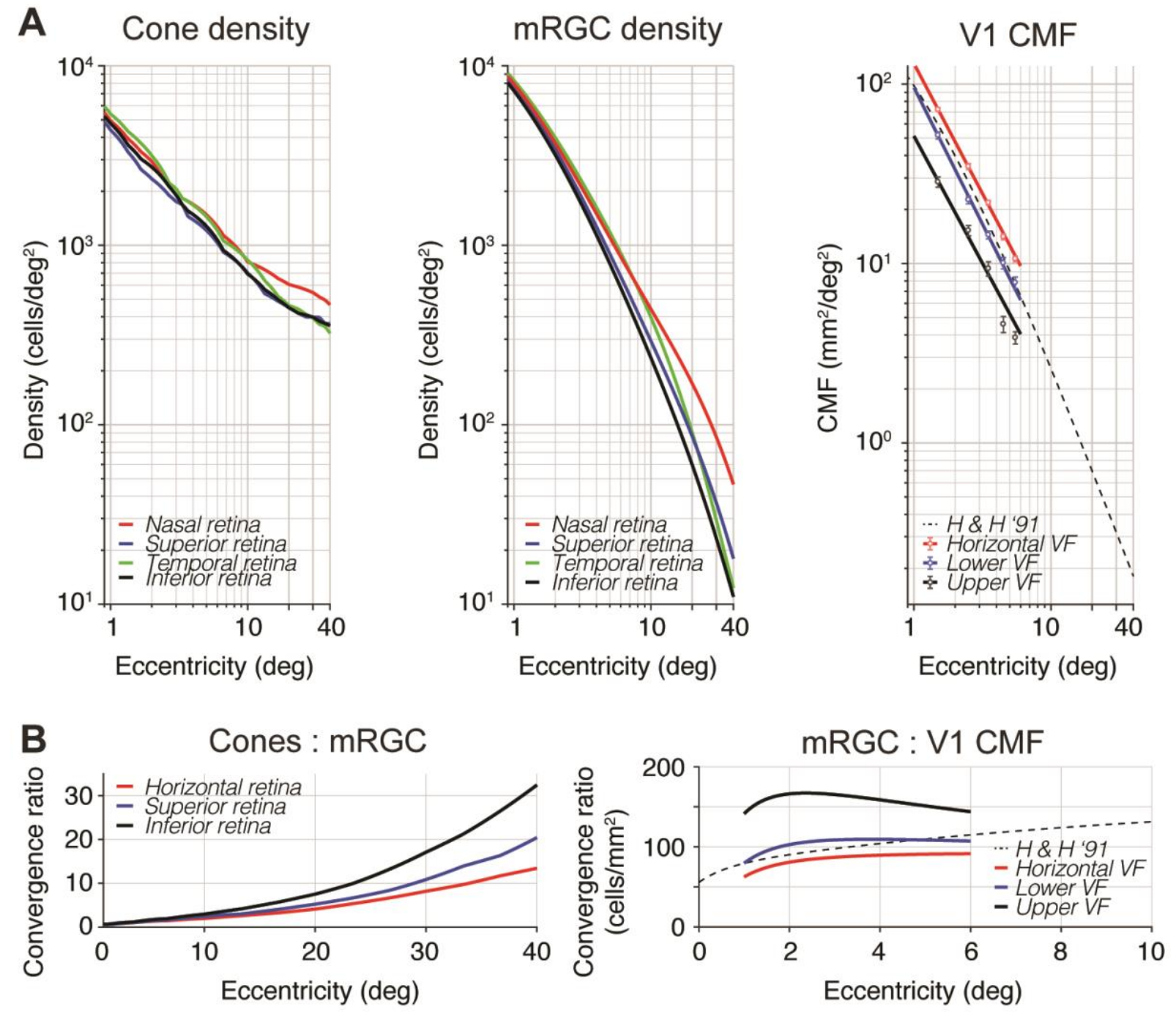

Fig 2. Nonuniformity in polar angle representations are amplified from cones to mRGCs to cortex. (A) Cone density, mRGC density, and V1-V2 CMF separate for cardinal meridians as a function of eccentricity. Left panel: Cone density from Curcio et al. [7]. Middle panel: mRGC densities are from Watson [60]. Both cone and $\mathrm{mRGC}$ data are in retinal coordinates from the left eye, where red, blue, green, and black lines represent nasal, superior, temporal and inferior retina computed with ISETBIO. Right panel: V1-V2 CMF computed from the HCP 7T Retinotopy dataset analyzed by Benson et al. [74] (color dots and lines) and predicted area CMF by the formula in Horton and Hoyt [61] (dashed black line, replotted from Fig 1). Both data types are plotted in visual field coordinates where red, blue and black data points represent the horizontal, lower and upper visual field meridians, respectively. Data points represent the median CMF of \pm 10 wedge ROls along the meridians for $1-6^{\circ}$ eccentricity in $1^{\circ}$ bins. Error bars represent 68\%-confidence intervals across 181 subjects using 1000 bootstraps. Colored lines are 1/eccentricity power functions fitted to data points. (B) Transformation ratios from cones to mRGCs and $\mathrm{mRGCs}$ to V1-V2 CMF. Ratios are shown separate for the horizontal (red), upper (blue) and lower (black) meridians. The cone:mRGC ratio is shown in retinal coordinates, with the horizontal meridian as the average of nasal and temporal retina. The mRGCs:V1 CMF panel is shown in visual coordinates and on a truncated x-axis due to the limited field-ofview during cortical measurements. These radial asymmetries are not depended on the type of displacement model used, as the general pattern can be found across two different computational RGC models (see Supplemental Fig 2 , second row).

Unlike the cones, $\mathrm{mRGC}$ receptive fields show a consistent asymmetry along the vertical meridian: The superior retinal meridian (lower visual meridian) contains a higher mRGC density 
compared to the inferior retinal meridian (upper visual meridian). This is consistent with the psychophysical VMA, showing better performance on the lower vertical meridian. This asymmetry increases with eccentricity. For example, the superior retina has $\sim 15 \%$ higher density compared to inferior at $3.5^{\circ}$, and $\sim 50 \%$ higher density at $40^{\circ}$ eccentricity. This interaction between retinal meridian and eccentricity is summarized in the cone-to-mRGC transformation plot, where the convergence ratio from cones to mRGCs increases more rapidly along the inferior retina than the superior and the horizontal retinal meridians (Fig 2A, middle panel).

\section{Radial asymmetries are amplified from mRGCs to early visual cortex}

Because the areal CMF calculation by Horton and Hoyt [61] does not make separate predictions for the cardinal meridians, we used the publicly available retinotopy dataset from the Human Connectome Project analyzed by Benson et al. [75] to calculate the CMF along the meridians (see also [74]). As a first check on agreement between the two datasets, we found that the V1V2 CMF data measured in 181 subjects with functional MRI [74], pooled across all polar angles, was a close match to Horton and Hoyt's [61] prediction based on lesion case studies from three decades ago. We then used the HCP dataset to compute CMF along the separate meridians.

We find that radial asymmetries in cortical magnification factors are yet larger than those found in mRGC density (Fig 2A, right panel), where V1-V2 CMF is higher on the horizontal than vertical meridian, and the V1-V2 CMF is higher for the lower than the upper vertical meridian. For example, at $3.5^{\circ}$ eccentricity CMF is $\sim 46 \%$ higher on the horizontal than vertical meridians and $\sim 46 \%$ higher for the lower vertical than upper vertical meridian. These radial asymmetries show a $2 x$ increase within the first three degrees of eccentricity before flattening and are mostly driven by the upper vertical meridian (Fig 2A, right panel). This indicates that the mapping of the visual field in early visual cortex is not simply predicted from the distribution of midget retinal ganglion cells, but rather the cortex amplifies the retinal radial asymmetries (Fig 2B, right panel).

\section{A computational observer model from stimulus to mRGCs to behavior}

To understand how radial asymmetries in visual field representations might affect visual performance, we added a retinal ganglion cell layer to our computational observer model [46]. In this observer model, we used the publicly available ISETBIO toolbox [69-71] to simulate the first stages of visual pathway including the stimulus scene, fixational eye movements, chromatic and 
achromatic optical aberrations, and isomerization by the cone array. Combining model output with a linear support vector machine classifier allowed us to simulate performance on a 2-AFC orientation discrimination task given information available in the cones. When matching stimulus parameters in the model to a previously published psychophysical experiment [11], we showed that biologically plausibly variations in optical quality and cone density together would contributed no more than $\sim 10 \%$ to the observed radial asymmetries in contrast sensitivity.

Given the inability of cone density to quantitatively explain differences in visual performance, we extended our model by adding spatial filtering properties of midget RGCs. We focus on mRGCs, because this processing stage shows a systematic asymmetry between the upper and lower visual field (where the cones did not), and an amplification of the horizontalvertical asymmetry. The $\mathrm{mRGC}$ computational stage is implemented after cone isomerizations and before the model performs the discrimination task. We provide a short overview of the modeled stages that precede the mRGC layer, as details of these stages can be found in our previous paper [46], followed by a discussion of the implementation details of the mRGC layer.

\section{Scene radiance}

The first stage of the model comprises the photons emitted by a visual display. This results in a time-varying scene defined by the spectral radiance of an achromatic low contrast Gabor stimulus (Fig 3, panel 1). The Gabor was oriented $15^{\circ}$ clockwise or counter-clockwise from vertical with a spatial frequency of 4 cycles per degree. These stimulus parameters were chosen to match a recent psychophysical experiment [28] to later compare model and human performance.

\section{Retinal irradiance}

The second stage simulates the effect of emitted photons passing through the human cornea, pupil and lens. This computational step results in time-varying retinal irradiance (Fig 3, second panel). Optics are modeled as a typical human wavefront with a 3-mm diameter pupil and contain a spectral filter that reduces the fraction of short wavelengths (due to the lens).

\section{Cone absorptions}

The third stage implements a rectangular cone mosaic with L-, M- and S-cones $\left(2 \times 2^{\circ}\right.$ field-ofview). For each cone, we compute the number of photons absorbed in each 2-ms bin, resulting in a $2 \mathrm{D}$ time-varying cone absorption image (Fig 3, third panel). The number of absorptions 
depends both on the efficiency of each cone type and on the wavelengths of light and on Poisson sampling due to the quantal nature of light. The cone absorptions include a second source of noise: fixational eye movements. We implemented two types of fixational eye movements, drift and microsaccades, using a model available in ISETBIO combining a statistical model of drift [76] with microsaccade statistics [77, 78]. This model shifts the stimulus across the cone array during the trial, where the retinal displacement is relatively small (about 2-4 cones within a trial at $4.5^{\circ}$ eccentricity). 


\section{Scene radiance

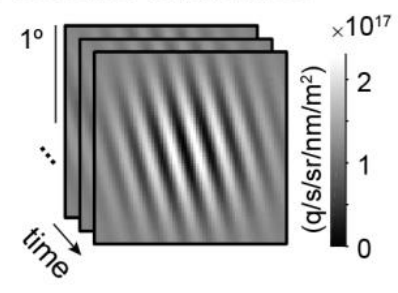

2. Retinal irradiance

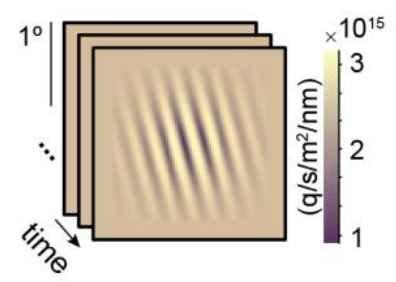

3. Cone absorptions

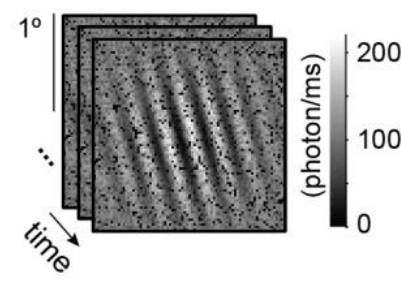

4. RGC responses

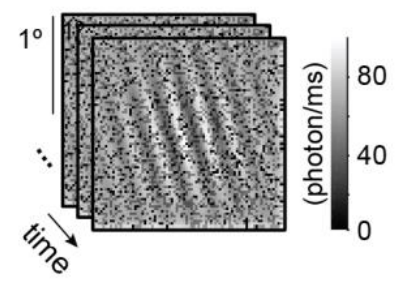

\section{Behavioral inference}

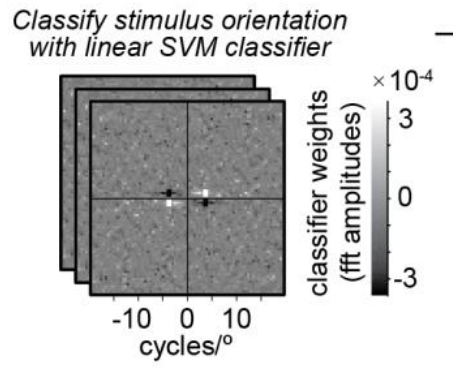

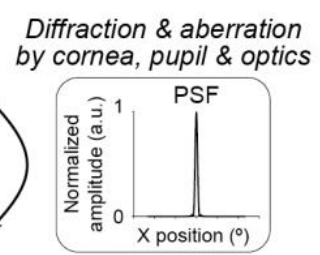
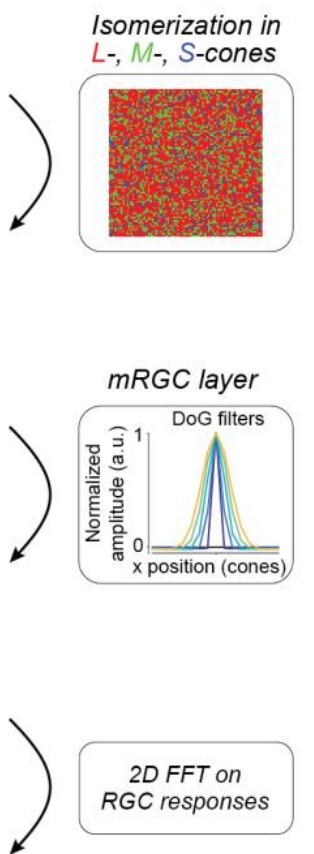

$\longrightarrow$ Calculate accuracy, fit Weibull, get contrast threshold

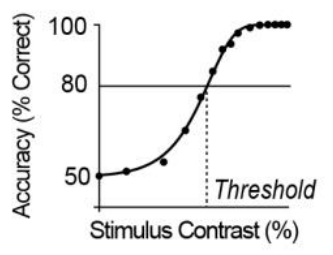

Fig 3. Overview of computational observer model with additional mRGC layer. A 1-ms frame of a $100 \%$ contrast Gabor stimulus is used at each computational step for illustration purposes. (1) Scene radiance. Photons emitted by the visual display, resulting in a time-varying scene spectral radiance. Gabor stimulus shows radiance summed across 400-700 nm wavelengths. (2) Retinal irradiance. Emitted photons pass through simulated human cornea, pupil, and optics, indicated by the schematic point spread function (PSF) in the top right-side box, resulting in timevarying retinal irradiance. Gabor stimulus shows irradiance with wavelengths converted to RGB values for illustration purposes. (3) Cone absorptions. Retinal irradiance is isomerized by a rectangular cone mosaic containing L-, Mand S-cones (red, green and blue pixels in box), resulting in time-varying photon absorption rates for each cone. This stage implements two noise sources: Poisson noise to capture photon noise and fixational eye movements by shifting the stimulus across the cone array during a trial. (4) RGC responses. Time-varying cone absorptions are convolved by a $2 \mathrm{D}$ difference of Gaussian model (DoG) to simulate band-pass filtering and subsampling by on- and off-midget 
RGCs (see also Fig 4). (5) Behavioral inference. Time-varying RGC responses are transformed to the Fourier domain using 2D FFT and phase information is removed. Without this transformation the classifier performs at chance level, as the individual pixel values are not informative for the task. This step provides the classifier the information that orientation information, but not phase information, is important for this task. A linear support vector machine (SVM) classifier is trained on FFT amplitudes to classify stimulus orientation per contrast level. Classifier weights averaged across time show peak amplitudes at peak spatial frequency of the stimulus (4 cycles/degree). With 10 -fold cross-validation, left-out data are tested, and accuracy is fitted with a Weibull function to extract the contrast threshold at $\sim 80 \%$.

\section{Midget RGC responses}

We add a rectangular retinal ganglion cell layer to capture the spatial filtering properties of midget RGCs. This computational stage is a simplified RGC model, solely capturing spatial filtering properties of retinal ganglion cells, and does not include a temporal filter, spatial subunits, or stochasticity in spike timing. We will elaborate on the possible contribution of other $\mathrm{RGC}$ properties to radial asymmetries in the Discussion.

The mRGC layer has the same field-of-view as the cone array. Because we do not model rectification or spiking non-linearities, we do not separately model on- and off-cells. Our $m R G C$ receptive fields are approximated as 2D difference of Gaussian (DoG) models [79, 80] (Fig 3, fourth panel), based on parameters from [81]. The width of the center Gaussian ( $\sigma_{c}, 1$ $\mathrm{sd}$ ) is $1 / 3$ of the spacing between neighboring $\mathrm{mRGC}$ receptive fields, and the surround Gaussian $\left(\sigma_{s}\right)$ is $6 x$ the width of the center. This creates an mRGC array where RFs overlap at 1.3 standard deviations from their centers, which matches the overlap of dendritic fields reported in human retina [50]. We compute the $\mathrm{mRGC}$ responses by convolving the cone absorptions with the $\mathrm{mRGC}$ DoG receptive field, and then by subsampling to get the correct $\mathrm{mRGC}$ density (Fig 4). The $m R G C$ density is determined by the cone array density and the cone-to-mRGC ratio, which we vary across simulations.

We can express the $\mathrm{mRGC}$ receptive fields in stimulus units (degrees of visual angle) because their inputs, the cones, are also defined in stimulus units. For a cone density typical of $4.5^{\circ}$ eccentricity on the horizontal meridian, a ratio of $1: 1 \mathrm{mRGC}$ to cones is best tuned to our stimulus spatial frequency, 4 cycles per degree (red dashed line, Fig 4B). 
A 1D representation in visual space

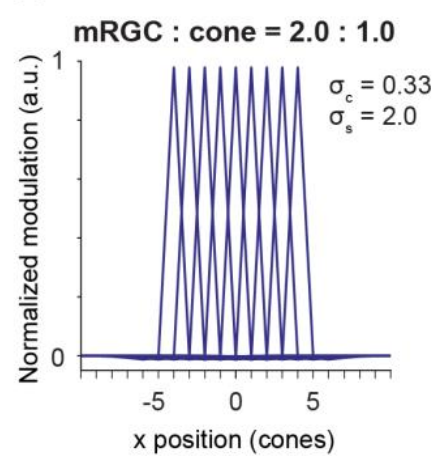

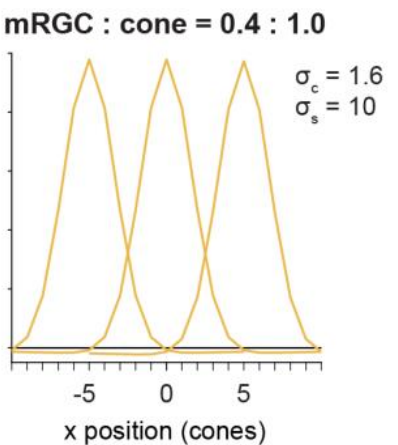

B 1D representation in Fourier space

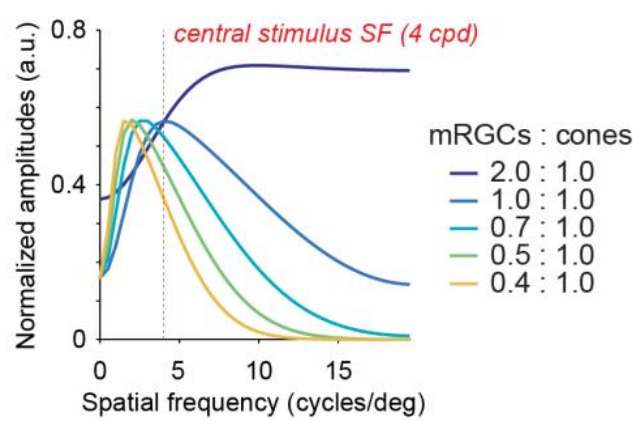

Fig 4. Difference of Gaussians filters used to model mRGC layer. Both 1D representations are illustrated for a $2 \times 2^{\circ}$ field-of-view $\mathrm{mRGC}$ array centered at $4.5^{\circ}$ eccentricity. (A) 1D representation of two example mRGC layers in visual space. The $m R G C$ responses are computed by convolving the cone image with the mRGC DoG RF, followed by subsampling the cone array to the corresponding mRGC density. Width for Gaussian center $\left(\sigma_{c}\right)$ and surround $\left(\sigma_{s}\right)$ are in units of cone spacing, which determines the subsample ratio to match the DoG width. Dark purple and yellow color of DoGs correspond to spectra in panel B. (B) 1D representation of Difference of Gaussians in Fourier space. The Fourier representation illustrates the band-pass and unbalanced nature of the DoGs (i.e. non-zero amplitude at DC). Depending on the width/subsample rate, DoGs attenuate different spatial frequencies. Peak stimulus frequency (4 cycles per degree) is indicated with red dashed line to illustrate what DoGs filters contain a peak frequency that would preserve most stimulus information. Fourier amplitudes are normalized.

\section{Behavioral inference}

The final stage of the computational observer model is the decision maker. For the main analysis, we use a linear support vector machine (SVM) classifier to discriminate if a stimulus orientation is clockwise or counter-clockwise from vertical given the RGC responses. As in our previous model [46], we apply a 2D fast Fourier transform to the RGC responses at each time point, and remove phase information. Classifying the mRGC amplitudes, rather than the full Fourier representation, allows the inference engine to learn a stimulus template that is robust to small eye movements and stimulus phase, and to categorize the stimulus orientation (illustrated by the high values at the stimulus spatial frequency (Fig 3, panel 5). The classifier trains and tests for each stimulus contrast separately, where each contrast level results in a percent correct identified stimulus. These accuracy results are fitted with a Weibull function to extract the contrast threshold at $\sim 80 \%$.

\section{Inference engine has a large effect on model performance}

We first ran a simplified version of our simulations in which the performance expectations were more straightforward to predict. We ran this version with three different observer models: an ideal observer, an observer based only on the signal-to-noise (SNR) level, and a linear SVM classifier. The simplified model has a cone mosaic with a single cone type (L-cone), does not include fixational eye movements, and does not include phase randomization of the stimulus. In 
this simplified model, the only source of noise is the Poisson process of photon absorption. This contrasts with the full model, which is too complicated to solve analytically, hence the use of a linear SVM classifier. In the simplified model, we can solve the closed-form analytical solution for the ideal observer as described by Geisler [82].

The ideal observer provides an upper bound on performance. This is because the observer has complete knowledge about the stimulus template and the noise distribution. As expected, our ideal observer model performs best of all decision makers with threshold values less than $0.1 \%$ contrast (Fig $\mathbf{5 A}$ ). These contrast thresholds are much lower (i.e. better performance) than those of human observers, who have thresholds of about $2-4 \%$ contrast for stimuli presented at the same eccentricity [28]. Given a fixed set of outputs from the cone mosaic, the ideal observer performance depends on the mRGC:cone ratio. The ideal observer performs best with an $\mathrm{mRGC}$ :cone ratio of 1:1, and more poorly with either a higher or lower ratio. The critical factor is not the number of $m R G C s$, but the receptive field properties. By design, the RF is scaled by the mRGC spacing, which in turn is determined by the combination of cone density and $\mathrm{mRGC}$ :cone ratio. At a 1:1 ratio for our cone mosaic and stimulus, the mRGC filter is best matched to the stimulus, as both peak at about 4 cycles per degree (Fig 4B). 
bioRxiv preprint doi: https://doi.org/10.1101/2020.10.20.347492; this version posted October 21, 2020. The copyright holder for this preprint (which was not certified by peer review) is the author/funder, who has granted bioRxiv a license to display the preprint in perpetuity. It is made available under aCC-BY-NC-ND 4.0 International license.

A

Ideal observer
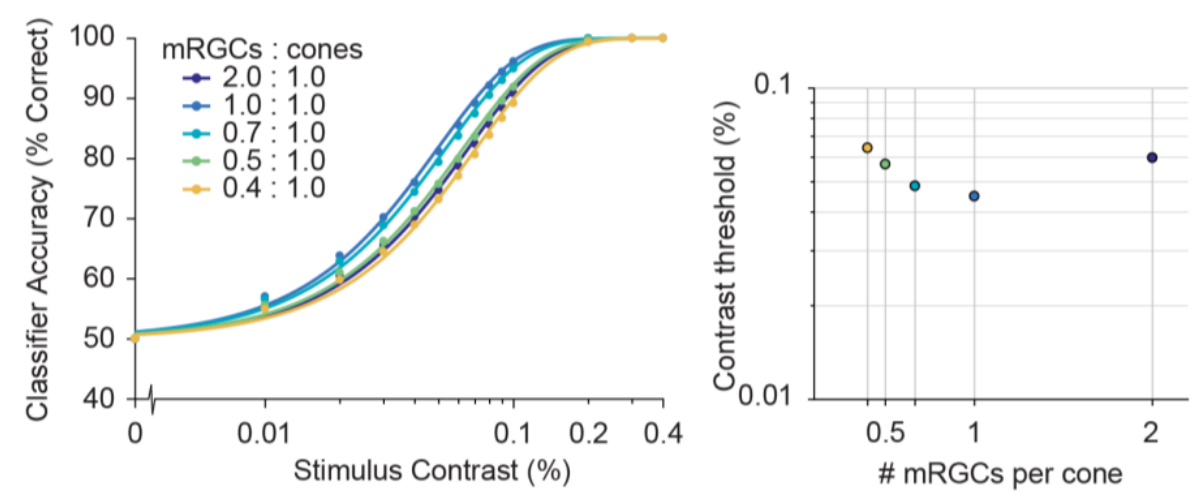

B
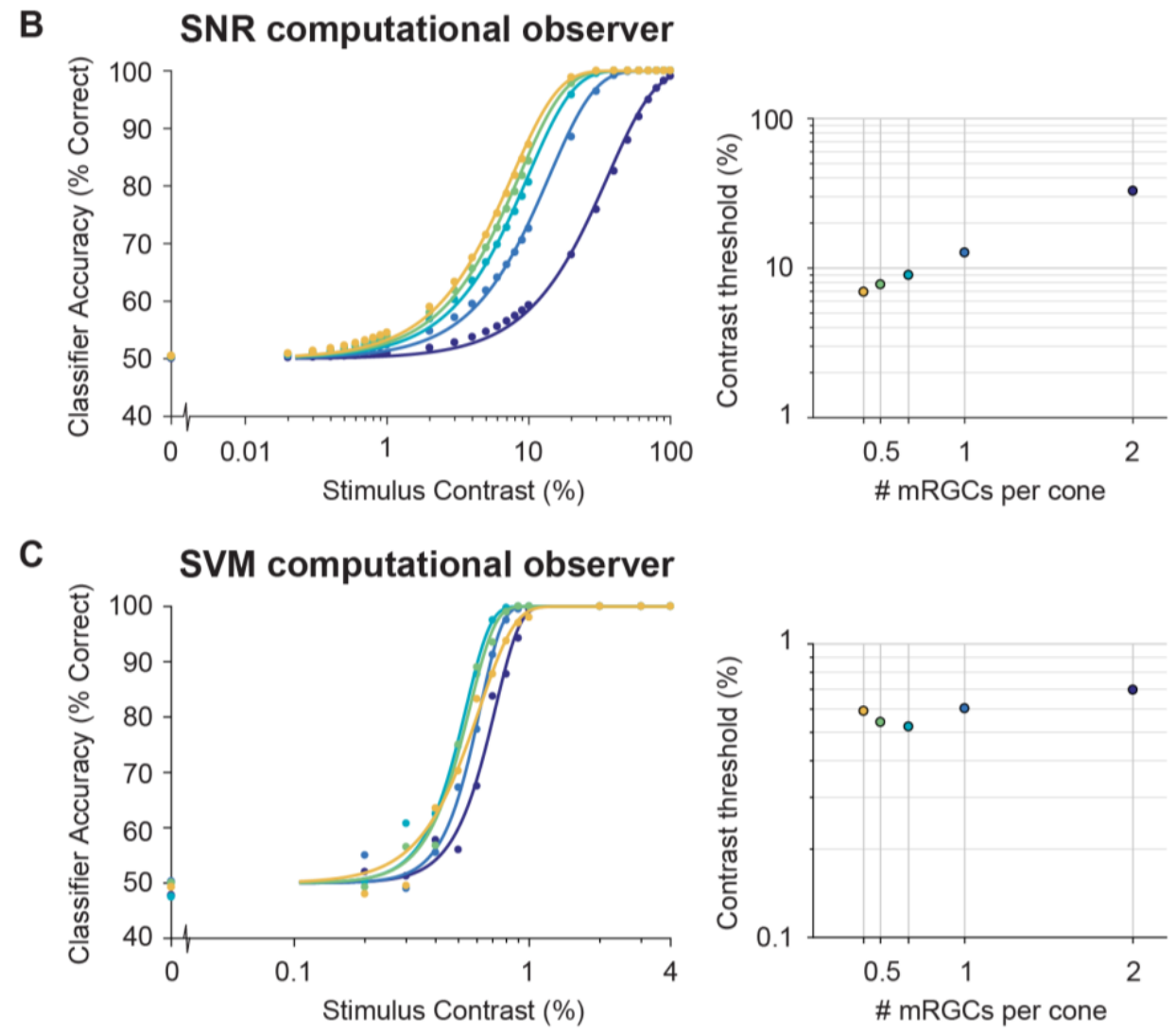

Fig 5. Model performance for different decision makers with an L-cone only mosaic. Left column shows classifier accuracy as function of stimulus contrast. Data are from one experimental iteration ( 200 trials per stimulus class) using a simplified version of the model with L-cone only mosaic at $4.5^{\circ}$ eccentricity, no fixational eye movements nor stimulus phase shifts. Data are fitted with a Weibull function. Contrast thresholds are plotted separately for each mRGC:cone ratio in the right column. (A) Ideal observer. The ideal observer computation follows Geisler [82], providing an upper bound on performance. (B) SNR computational observer. This decision maker provides a lower bound on performance. Signal is defined as the difference in power between noiseless clockwise and counter-clockwise mRGC responses. Noise is defined as the power across the background (noise-only) mRGC responses. (C) SVM computational observer. Performance of the SVM observer is in intermediate of the SNR and ideal observer. This is because the SVM observer learns a stimulus template but is limited by the number of trials. 
Second, we computed performance based on discriminability of the mRGC outputs for an observer with no knowledge of the stimulus classes, the "SNR observer". The SNR observer cannot classify individual stimuli as 'clockwise' or 'counter-clockwise' because it has no knowledge of the stimulus classes, but it can classify a pair of stimuli as same or different. The same/different judgment depends only on how different the mRGC outputs are for a stimulus pair, operationalized as the total power in the difference image between the mRGC outputs for the two Gabor stimuli. This operation is equivalent to putting equal classifier weights across all mRGCs. This contrasts with the ideal observer, which weights locations based on how informative they are.

Specifically, we first compute the SNR in the following way. We define the signal as the total power in the mRGC difference image for noiseless stimuli from the two classes. We define the noise as the total power across the background (noise-only) image. The intuition is that if the mRGC outputs for the stimulus pairs (clockwise vs counter-clockwise) differ a lot compared to the level of background noise, the SNR is high, and the stimuli are discriminable. The discriminability index (d') can be computed from the SNR, and from d' we derive the percent correct (assuming no bias). This enables us to analyze the SNR model the same way as the ideal observer and the SVM classifier.

As expected, the SNR observer performs worse than human observers and the ideal observer because it has no knowledge of the stimulus classes. The average thresholds for the SNR observer are around 10\% contrast (Fig 5B). The pattern of performance as a function of mRGC:cone ratios differs from the ideal observer. For the SNR observer, performance is best with the smallest ratio of $\mathrm{mRGCs}$ to cones, and systematically declines with increasing ratio. This might seem surprising, as the smallest ratio means the fewest mRGCs. The better performance is due to the receptive field properties, not to the low cell density per se. The model with the smallest mRGC:cone ratio, by design, has the largest receptive fields, which are tuned to low frequencies. These low-frequency filters remove much of the background noise, increasing the SNR.

Third, we computed performance from an SVM computational observer model. This model performs intermediate between the ideal and the SNR observer, both in overall accuracy and in the pattern across cell densities. This is because the SVM observer has more information about the stimulus than the SNR observer, but still performs worse than an ideal observer as it has to learn the stimulus template from limited data (in this case 200 trials per stimulus class). Contrast thresholds are on average about $0.6 \%$, lower than the SNR model and human 
performance, and higher than the ideal observer model (Fig 5C). The performance pattern across different $\mathrm{mRGC}$ :cone ratios is also intermediate between the ideal and the SNR models, performing best with the third filter (ratio of 0.7 mRGCs per 1 cone). If the SVM observer received more training trials, it would perform closer to the ideal observer, whereas fewer trials would make it perform more like the SNR observer.

\section{The effect of spatial filtering by mRGCs on orientation discrimination}

For the previous analyses, we assumed a fixed cone density and varied the mRGC density. Because both densities vary with cardinal meridians in the human retina, we considered how performance depended on the two factors together. Moreover, we implemented a more complete simulation, including fixational eye movements, phase randomization of the stimulus, and a trichromatic retina. For this more complex simulation, we implemented the linear SVM computational observer, as closed form solutions are unwieldy for the ideal observer and SNR observer model.

For comparison with our observer model based on cone outputs in our prior work [46], we used the same simulations as previously (identical eye movement patterns, cone densities, L-, M-, S-cone distributions), and to each of these simulations we added one of five mRGC layers, varying in density and filter widths.

As with the models based on cone outputs, we find that models based on mRGC outputs have better performance (lower contrast thresholds) when cone density is higher. The slope of this function depends on the mRGC:cone ratio, where lower ratios result in steeper functions (Fig 6A). This indicates that performance depends on both mRGC density and cone density. To examine the space of predicted contrast thresholds as a function of cone density and $\mathrm{mRGC}$ :cone ratio, we plot performance as a function of both variables with linear interpolation (Fig 6B). This 3D mesh shows best performance (lowest thresholds) at intermediate $\mathrm{mRGC}$ :cone ratios and high cone densities. As the cone density decreases, best performance shifts to higher mRGC:cone ratios. This pattern indicates that the highest cone densities and narrowest spatial filters do not necessarily result in best performance (i.e. the lowest contrast thresholds), but rather performance depends on the interaction between the stimulus spatial frequency and what signal/noise components are attenuated by spatial filters. 
bioRxiv preprint doi: https://doi.org/10.1101/2020.10.20.347492; this version posted October 21, 2020. The copyright holder for this preprint (which was not certified by peer review) is the author/funder, who has granted bioRxiv a license to display the preprint in perpetuity. It is made available under aCC-BY-NC-ND 4.0 International license.

A

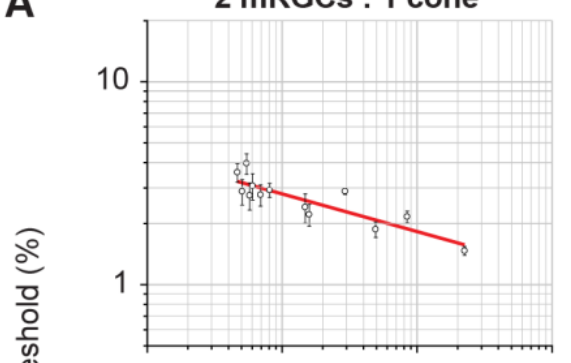

0.5 mRGC : 1 cone

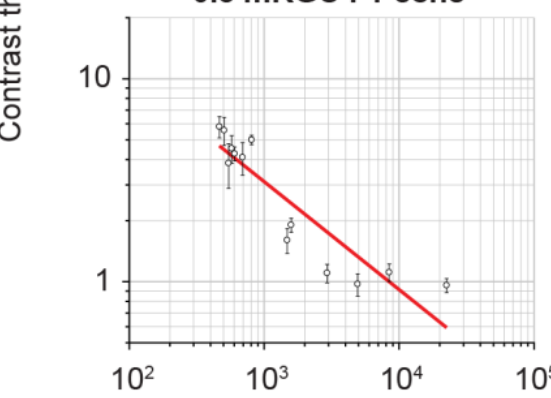

\section{B}

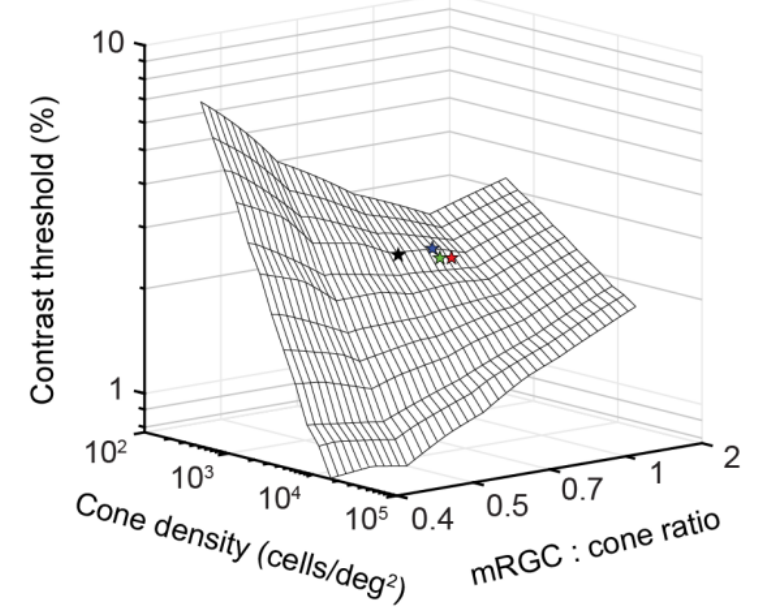

1 mRGC : 1 cone

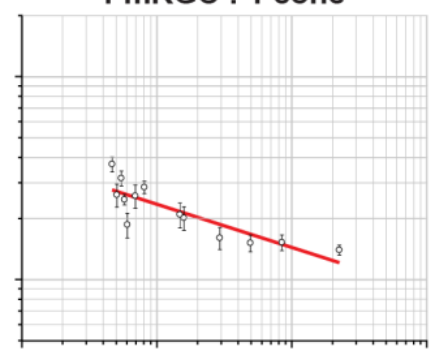

0.4 mRGC : 1 cone

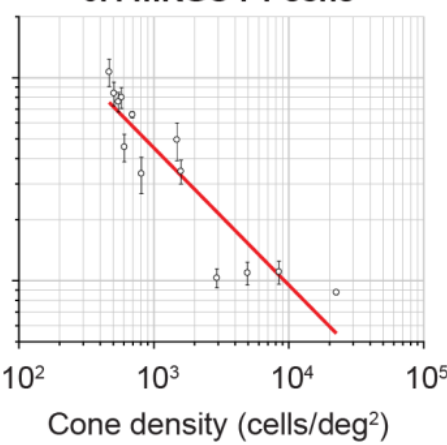

0.7 mRGC : 1 cone

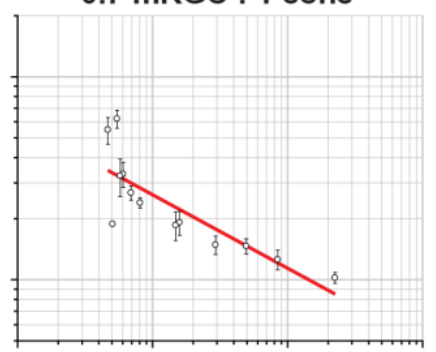

All 5 mRGCs : cone ratios

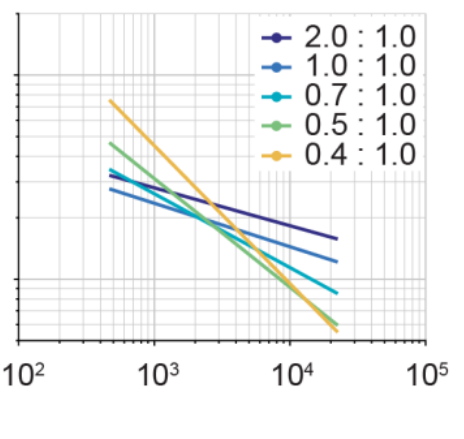

Radial locations at $4.5^{\circ}$ eccentricity:

$\star$ Nasal retina $\hbar$ Temporal retina

$\star$ Superior retina $\star$ Inferior retina

Fig 6. The effect of spatial filtering properties by mRGCs on full model performance. (A) Contrast thresholds as a function of cone density and mRGC:cone ratio. Panels with data points and power law fit (red line) show contrast thresholds for each mRGC:cone ratio separately. All 5 fits are replotted in the right bottom panel. Data are averaged across 5 iterations of a simulated experiment varying in cone density. Error bars represent the standard error from bootstrapping the mean across 5 iterations, 1000 times with replacement. Goodness of fit for individual power law are $R^{2}=0.75, R^{2}=0.73, R^{2}=0.68, R^{2}=0.83, R^{2}=0.85$, for the highest to lowest mRGC: cone ratios respectively. (B) Two views of combined effect of cone density and mRGC:cone ratio on contrast sensitivity. The 3D mesh is built by ordering individual contrast threshold fits ( $y$-axis) by corresponding cone density ( $x$-axis) and mRGC:cone ratio (z-axis). The mRGC:cone ratios are up sampled by a factor of 8 and cone density is resampled to have equal log10 spaced values. Individual dots represent the predicted model performance for nasal (red star), superior (blue star), temporal (green star) and inferior (black star) retinal locations at $4.5^{\circ}$ eccentricity (matched to stimulus eccentricity in [28]). 


\section{Comparison between model and human contrast sensitivity}

To compare model performance to human observers, we first quantify the range of biologically plausible cone densities and mRGC:cone ratios at $4.5^{\circ}$ eccentricity and the four cardinal meridians. This eccentricity is used to match stimulus parameters from a recent psychophysical experiment [28].

According to Curcio et al. [7], cone density varies at $4.5^{\circ}$ eccentricity from $\sim 1,575$ cones $/ \mathrm{deg}^{2}$ on the horizontal retinal meridian (nasal: 1590 cones $/ \mathrm{deg}^{2}$, temporal: 1560 cones $/ \mathrm{deg}^{2}$ ), to 1300 cones $/ \mathrm{deg}^{2}$ on the superior retinal meridian, and $1382 \mathrm{cones} / \mathrm{deg}^{2}$ on the inferior retinal meridian. We combine these cone density values with the mRGC:cone ratios from the computational model by Watson [60], which ranges between $0.84: 1.0$ on the horizontal meridian (nasal: $0.87: 1.0$, temporal: $0.82: 1.0$ ), to $0.81: 1.0$ on the superior retina and $0.68: 1.0$ on the inferior retina.

Across these ranges, there is only slight performance differences in our computational observer model (Fig 6B, 4 dots on the 3D mesh). The overall performance within this range is similar to human observers, with contrast sensitivity of about $40 \%$ (Fig 7A, compare middle vs right panel). Moreover, the pattern of performance across the polar angles is qualitatively similar to human behavior, with better performance on the horizontal than the vertical meridian, and slightly better performance on the lower vertical meridian than the upper vertical meridian. However, the asymmetries are not quantitatively similar to human performance, which varies far more as a function of meridian.
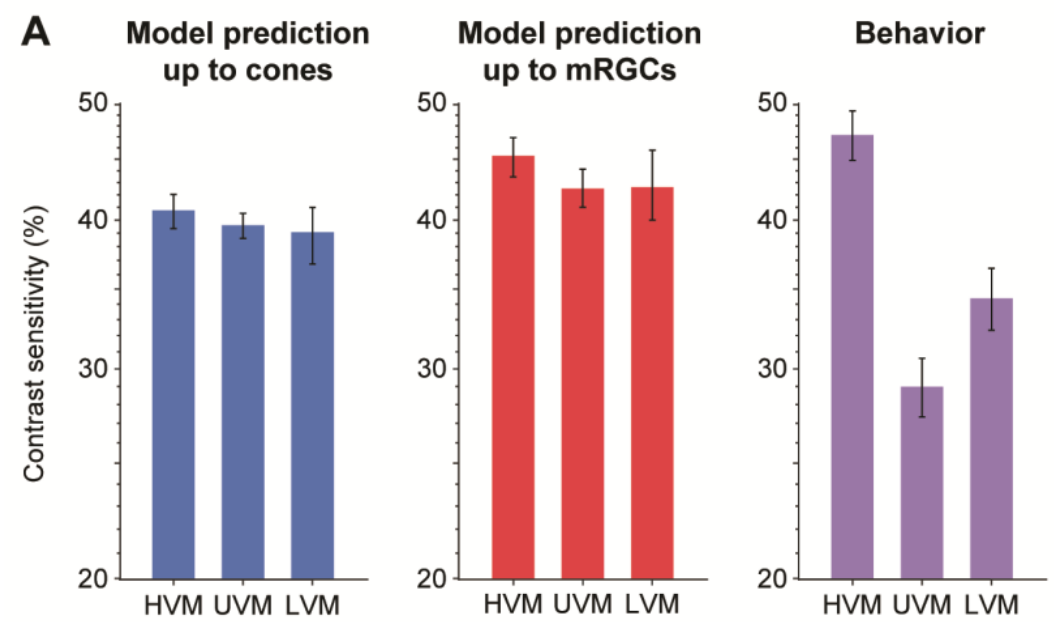

B Radial asymmetries

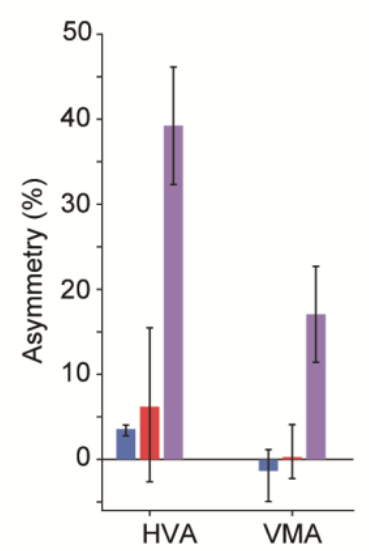

Fig 7. Comparison of model performance to human performance. (A) Contrast sensitivity predicted by computational observer model up to cones, up to mRGCs, and behavior using matching stimulus parameters. HVM: horizontal visual meridian, UVM: upper visual meridian, LVM: lower visual meridian. Model prediction shows contrast sensitivity (reciprocal of contrast threshold) for stimuli at $4.5^{\circ}$ eccentricity, with a spatial 
frequency of 4 cycles per degree. HVM is the average of nasal and temporal meridians. Y-axis is truncated at $20 \%$ contrast sensitivity. Model error bars indicate simulation results allowing for uncertainty in the cone or mRGC density along each meridian (See Methods for details). Behavioral plots show group average results $(n=9)$ from Himmelberg et al. [28], and error bars represent standard error of the mean across observers. (B) Radial asymmetries for cones, mRGCs and behavior. HVA: horizontal-vertical asymmetry. VMA: vertical-meridian asymmetry. Blue, red and purple bars match panel (A) and correspond to model prediction up to cones, mRGCs, human behavior. Error bars represent the HVA and VMA when using the upper/lower bound of predicted model error from panel A.

To summarize the model and human performance, we calculate the percent change in horizontal vs vertical meridian (HVA) and upper vs lower vertical meridian (VMA). We find that, although $\mathrm{mRGC}$ density amplifies the radial asymmetries present in the cones, these biological variations only predict a horizontal-vertical asymmetry of about $6.3 \%$ (Fig 7B, red bars). This is about double compared to a model without the mRGCs (3.4\%, Fig 7B blue bars) (see also Supplemental Fig 3). For the vertical-meridian asymmetry, the predicted asymmetry of the mRGC model is $0.35 \%$, much smaller than that of human performance (17\%) [28]. Although far from human performance, it is better than a model that does not include the mRGCs, which actually predicts the opposite asymmetry to behavior: $-1.6 \%$ as cone density is higher for the upper vertical meridian than the lower vertical meridian.

The higher overall contrast sensitivity in the mRGC model compared to the cone model might at first seem counter-intuitive, as successive stages of processing can only lose information. This intuition holds for ideal observers. However, our models are computational observers, not ideal observers, and use a linear SVM classifier that learn weights from the simulated responses. Using such an SVM classifier to make behavioral inferences causes the absolute performance to depend on factors such as the efficiency of learning the stimulus template and number of trials. If we had conducted more simulations, overall performance would have improved. However, we are more interested in the differences in performance across cardinal meridians than in the absolute performance. Importantly, our modeling approach suggests that although including an mRGC layer predicts radial asymmetries closer to behavior than a model up to the cones, the biological variations in the spatial properties of $\mathrm{mRGCs}$ are far from sufficient to fully explain differences in behavior. 


\section{Discussion}

The visual system, from retina to subcortex to cortex, is organized in orderly maps of the visual field. But within each particular processing stage, the retinotopic map is distorted. Here we investigated the radial asymmetries in these spatial representations across three stages of the early visual pathway: cones, mRGCs and V1-V2 cortex. Our study revealed that both the eccentricity gradient (foveal bias) and radial asymmetries (HVA and VMA) in spatial representations are amplified from cones to mRGCs, and further amplified from mRGCs to early visual cortex. Additionally, we showed that although $\mathrm{mRGC}$ density has radial asymmetries in the directions predicted by psychophysical studies, they are insufficient to explain most of the differences in human's contrast sensitivity around the visual field.

\section{Linking behavior to eccentricity and radial asymmetries in visual field representations}

For more than a century, limits in retinal sampling were hypothesized to cause the fovea-toperiphery gradient in human visual performance $[1,5,6]$. Initial tests of this idea showed that the fall-off in cone density could explain some, but not all of the observed decrease in visual acuity [2, 3, 83-86]. Later, more detailed computational models, reported that mRGCs come closer in predicting the eccentricity-dependent decrease in achromatic contrast sensitivity or resolution, and conclude that mRGCs are sufficient in explaining some aspects of behavior, especially spatial resolution and contrast sensitivity [87-93]. Similar to the retina, the cortical magnification factor in V1 has been linked to visual performance as a function of eccentricity, for example, explaining differences in acuity [91, 94, 95], contrast sensitivity and resolution [16], and the strength of some visual illusions [96].

Conversely, radial asymmetries have often not been considered. For instance, all abovementioned studies either ignored the stimulus polar angle for analysis or limited measurements to a single meridian, usually the horizontal. Despite the fact that the existence of radial asymmetries in human early visual cortex was predicted based on behavior in the late 70's [15, 16], further reports on radial differences have been scarce. One fMRI study reported a difference in V1 BOLD amplitude for stimuli on the lower and the upper visual meridian [97] and two studies found more cortical surface area devoted to the horizontal than the vertical meridian $[98,99]$. Yet several studies have assumed little to no radial differences in macaque V1 CMF $[100,101]$ or did not account for radial differences in human V1 CMF $[44,95]$ to explain 
differences in behavior. Computational models that include retinal and/or V1 sampling across visual space generally exclude radial asymmetries (e.g. [102, 103]). A few cases do incorporate radial asymmetries in the retinal ganglion cell distribution, but they assume that these asymmetries are not further amplified in cortex [104-106].

\section{Early visual cortex does not sample the retina uniformly}

It is well documented that the convergence of cones to retinal ganglion cells varies with eccentricity (e.g. see [90]). In the fovea of both primates and humans, there is one cone per pair of bipolar cells and pair of midget RGCs, with pairs comprised of an "on" and an "off" cell. In contrast, in the periphery, there are many cones per pair of bipolar cells and midget RGCs, with the ratio depending on the eccentricity. In the far periphery, there can be dozens of cones per ganglion cell [7].

It has been long debated whether V1 further distorts the visual field representation, or if V1 samples uniformly from RGCs, as reviewed previously [64, 65]. Our analysis showed more cortical surface area devoted to the fovea than the parafovea and the horizontal than vertical meridian, supporting previous findings using retinotopy informed by anatomy [98] and functional MRI [74, 99]. Importantly, these eccentricity and radial non-uniformities are larger in V1 than expected from asymmetries in $\mathrm{mRGC}$ density projected to cortex, confirming histology studies in monkey [56, 66-68, 107, 108]. Whether these non-uniformities arise in cortex, or depend on the mapping from retina to LGN, LGN to V1, or both, is a question of interest in both human [109, 110] and monkey [111-115], but beyond the scope of this paper. The implication is that cortex cannot be understood as a canonical wiring circuit from the retina repeated across locations.

Because visual field distortions are larger as a function of eccentricity than polar angle, one might surmise that radial asymmetries contribute little to visual performance. Even though the polar angle asymmetries are smaller than the eccentricity effects, they can in fact be large. For example, within the central eight degrees, the surface area in $\mathrm{V} 1$ is about $60 \%$ larger for the horizontal meridian than the vertical meridian [74]. Given that virtually all visual tasks must pass through V1 neurons, these cortical asymmetries are likely to have a large effect on perception. The number of cortical cells could be important for extracting information quickly [116], for increasing the signal-to-noise ratio, and for tiling visual space and visual features (e.g., orientation, spatial frequency) more finely [117]. To know how the number of V1 neurons affect performance, there is a need for a computational model that explicitly links cortical resources to performance around the visual field. 


\section{Model limitations}

Despite implementing known facts about the eye, our model, like any model, is a simplification. Some of the simplifications allow for interpretable results. For this model, we make the trade-off between complexity and understanding by treating a local patch of mRGCs as a linear, shiftinvariant system (i.e., a spatial filter). As the model here is identical to our previous model, except for the mRGC layer, we will focus on the limitations of this mRGC layer, and refer to Kupers, Carrasco and Winawer [46] for model limitations related to the pathways from scene to cone absorptions and the inference engine.

\section{Uniform sampling within a patch}

The $\mathrm{mRGC}$ layer is implemented as a rectangular patch of retina, initially with the same size as the cone mosaic. This allows for filtering by convolution and then linear subsampling to account for mRGC density, making the model computationally efficient. We do not incorporate two complexities of RGC sampling in the retina: (i) density gradients within a patch and (ii) irregular sampling. (i) Given our relatively small patch size $\left(2 \times 2^{\circ}\right.$ field-of-view) in the parafovea (centered at $\left.4.5^{\circ}\right)$, the change in density across the patch would be small $(\sim 10 \%)$. We found that a much larger change in $\mathrm{mRGC}$ density (spanning a 5-fold range) had only a modest effect on performance of our observer model, so it is unlikely that accounting for a small gradient within a patch would have significantly influenced our results. (ii) Given the relatively low spatial frequency content of our test stimulus, it is unlikely that irregular sampling would have resulted in a substantial difference from the regular sampling we implemented.

Nonetheless, it is important to assess the degree to which these complexities are expected to affect performance for other tasks, especially for large retinal patches and high spatial frequency stimuli. Hexagonal cone arrays that include within-patch density gradients have been implemented in ISETBIO by Cottaris et al. (see for example [118, 119]). Extending similar approaches to models of the RGC circuitry will allow for direct tests of the importance of the gradients and irregularities. For some tasks, they are likely to be important. When we expect them to have little impact, as here, we choose the more straightforward mathematical implementation.

\section{Linear spatial summation and no temporal summation}

Our mRGC layer modeled one property of RGCs: linear spatial summation. The summation was computed over the photon absorptions by the cones. We did not account for temporal 
summation, or for non-linearities in the integration of signals from cones to mRGCs. Temporal summation and non-linearities are known to occur both within the cones [120] and in the downstream retinal circuitry (e.g., [121, 122]). To our knowledge, none of these properties are known to vary systematically around the visual field. We focused on cell density and RF size because there is clear evidence that these properties vary across the meridians, and they do so in a manner correlated with psychophysical performance. We showed that this variation in physiology by itself does not account for most of the observed performance effects. Whether temporal summation and spatial non-linearities accentuate the differences caused by density variation is a question to be addressed in future work. One hint that combining multiple model components can lead to predicted patterns of behavior not expected from either of the model components separately is the case of eye movements and temporal integration. Eye movements without temporal integration poses only a minor challenge for decoders. But eye movements combined with temporal integration results in spatial blur, and degrades performance, causing a loss in contrast sensitivity up to a factor of 2.5 , with larger losses for stimulus spatial frequencies over 8 cycles per degree [119].

The discussion above raises the question, had we incorporated more features of the retina in our model, would the model make predictions more closely matched to human performance? We think this is unlikely, because we measured substantially larger asymmetries in cortex than in retina. If the retinal simulations entirely accounted for behavior, this would leave no room for the additional cortical asymmetries on behavior.

\section{Other retinal cell types}

Midgets are not the only retinal ganglion cells that process the visual field. Parasol ( $p R G C s$ ) and bistratified retinal ganglion cells are less numerous but also cover the entire retina. pRGCs are the next most common retinal ganglion cells, and have generally larger cell bodies and dendritic field sizes than mRGCs, both increasing with eccentricity [49]. These differences cause parasols to be more sensitive to relative contrast changes and have higher temporal resolution, with the consequence of losing spatial resolution [123]. For this reason, the small mRGCs are much more likely to put a limit on spatial vision, and thus our model does not include pRGCs. We did not include or quantify radial densities in other intermediate cell layers in the retina, such as bipolar cells, horizontal cells, or amacrine cells. Their contributions are likely to introduce additional non-linearities, but it is unknown if their properties contain any radial asymmetries, and thus, to our knowledge, have not been incorporated in models. 


\section{A case for cortical contributions to visual performance asymmetries}

Our model incorporating spatial properties of $\mathrm{mRGC}$ only explained a small amount of the differences in behavior. This indicates a contribution from downstream processing, such as early visual cortex. This contrasts with recent retinal modeling of contrast sensitivity in the fovea, for which very little information used for behavior seems to be lost from the retinal output [119]. This may not be the case for the parafovea and periphery. V1-V2 cortex has several characteristics that suggest a tight link between cortical topography and radial visual performance asymmetries. Hence a model that incorporates properties of early visual cortex is likely to provide a substantially better account of radial asymmetries in behavior than one that only incorporates properties of the eye. We have not developed such a model but outline some of the reasons that cortex-specific properties are important for explaining radial asymmetries.

First, the representation of the visual field is split across hemispheres in visual cortex along the vertical, but not horizontal meridian. This split may require longer temporal integration windows for visual input that spans the vertical meridian, as information needs to travel between hemispheres. For example, the response in the left visual word form area is delayed by $\sim 100$ ms compared to the right visual word form area when presenting a stimulus in the left visual field [124]. Longer integration windows may in turn impair performance on some tasks, as eye movements during integration will blur the representation. Longer integration time of visual information spanning the vertical meridian is consistent with behavior, as accrual time is slower when stimuli are presented at the vertical than the horizontal meridian [36].

Interestingly, the hemispheric split is not precise: there is some ipsilateral representation of the visual field along the vertical meridian in early visual cortex [125]. The amount of ipsilateral coverage is larger along the lower than upper vertical meridian and increases from 1 $6^{\circ}$ eccentricity. It is possible that the split representation affects performance for stimuli on the vertical meridian (contributing to the HVA), and that the asymmetry in ipsilateral coverage between the lower and upper vertical meridian contributes to the VMA.

Second, there is good correspondence between the angular patterns of asymmetries in V1-V2 cortex and behavior. Radial asymmetries in the CMF of early visual cortex are largest along the cardinal meridians (i.e. horizontal vs vertical and upper vertical vs lower vertical). The asymmetries gradually fall-off with angular distance from the meridians [74]. This gradual decrease in radial asymmetry in cortex parallels the gradual decrease in contrast sensitivity [10, $25,26]$ and spatial frequency sensitivity [32] with angular distance from the cardinal meridians. 
Measurements of cone density and retinal ganglion cell density have emphasized the meridians, so there is less information regarding how the asymmetries vary with angular distance from the meridians.

Third, there is good correspondence between cortical properties and behavior in the domain of spatial frequency. Radial asymmetries in spatial frequency sensitivity observed by Barbot et al. [32] parallel spatial frequency tuning in V1 cortex. Specifically, fMRI measurements show that in $\mathrm{V} 1$, the preferred spatial frequency tuning is higher along the horizontal meridian than vertical [126], and in behavior spatial frequency thresholds are higher on the horizontal than vertical [32].

Fourth, radial asymmetries in behavior are maintained when tested monocularly [10, 32], but thresholds are slightly higher compared to binocular testing (at least for spatial frequency sensitivity [32]). Higher thresholds (i.e. poorer performance) show that performance benefits from combining information of the two eyes, as twice the amount of information increases the signal-to-noise ratio [127]. This summation is likely to arise in early visual cortex, as V1 is the first stage in the visual processing pathways where information of the left and right visual field merges [128-130].

\section{Conclusion}

Overall, we have shown that the well documented radial asymmetries in visual performance are associated with differences in the structural organization of cells throughout the early visual pathway. Radial asymmetries in cone density are amplified in downstream processing, from cones to RGCs and again from RGCs to early visual cortex. Further, we have extended our computational observer model to include spatial filtering of mRGCs and found that its contributions, although larger than those of cones, is far from explaining behavior. Future research will aim to integrate cortical data within the computational observer model to explain whether a significant amount of the radial asymmetries can be accounted for by the organization of cortical space in early visual cortex. 


\section{Methods}

\section{Reproducible computation and code sharing}

All analyses were conducted in MATLAB (MathWorks, MA, USA). Data and code for our previously published and extended computational observer model, including density computations and figure scripts, are made publicly available via the Open Science Framework at the URL: https://osf.io/mygvu/ (previously published) and https://osf.io/d89s5/ (this study).

\section{Data sources}

Data on cone density, midget RGC density, and V1-V2 cortical surface area previously published or from publicly available analysis toolboxes. Both cone and mRGC densities were computed as cells $/ \mathrm{deg}^{2}$ for $0-40^{\circ}$ eccentricities (step size $\left.0.05^{\circ}\right)$, at the cardinal meridia $\left(0^{\circ}, 90^{\circ}\right.$, $180^{\circ}$ and $270^{\circ}$ polar angle, corresponding to nasal, superior, temporal and inferior retina of the left eye. Fig 1 contains averaged cone and mRGC densities across all meridians as a function of eccentricity.

\section{Cone density}

Cone density data for the main results were extracted from post-mortem retinal tissue of 8 human retina's published by Curcio et al. [7] using the analysis toolbox ISETBIO [69-71], publicly available via GitHub (https://github.com/isetbio/isetbio).

Cone density in Supplemental Fig 1 shows two datasets computed by two analysis toolboxes. To extract post-mortem data from Curcio et al. [7], we either use ISETBIO or the rgcDisplacementMap toolbox [72], publicly available at GitHub

(https://github.com/gkaguirrelab/rgcDisplacementMap). A second cone density dataset comes from an adaptive optics study published by Song et al. [8]. From this work, we use "Group 1" (young individuals, 22-35 years old) implemented in ISETBIO.

\section{Midget retinal ganglion cell receptive field density}

Midget RGC density for the main results were computed with the quantitative model by Watson [60] implemented in ISETBIO. This model combines cone density data from Curcio et al. [7], mRGC cell body data from Curcio \& Allen [48] and the displacement model by Drasdo et al. [52], to predict the midget $R G C$ receptive fields (RFs). 
Midget RGC data in Supplemental Fig 1 computes mRGC density with two computational models: Watson [60] from ISETBIO and the displacement model by Barnett \& Aguirre [72] implemented in the rgcDisplacementMap toolbox.

\section{Cortical magnification factor in early visual cortex}

To quantify the fovea-to-periphery gradient in the $\mathrm{V} 1$ cortical magnification factor (CMF), we used the areal CMF function published in Horton and Hoyt [61] for 0-40 eccentricity (Fig 1). Because the function by Horton and Hoyt does not make separate predictions for the cardinal meridians (Fig 2), we used data from the Human Connectome Project (HCP) 7 Tesla retinotopy dataset $(n=181)$. HCP data were first published by Ugurbil, van Essen, and colleagues [131, 132] and analyzed with population receptive field models by Benson et al. [75]). V1-V2 CMF surface area data are from Benson et al. [74] and computed as follows. (Note that more details on these methods are available Benson et al. [75]).

To compute V1-V2 CMF from retinotopy data, we used the extracted surface area in $\pm 10^{\circ}$ wedge ROls centered on the cardinal meridia in each individual's hemisphere. The wedges on the horizontal, dorsal, and ventral locations represented the horizontal, lower, and upper visual field meridians respectively. The wedges were separated into 5 bins between $1-6^{\circ}$ eccentricity $\left(1^{\circ}\right.$ step size, i.e. $\left.1-2^{\circ}, 2-3^{\circ}, 3-4^{\circ}, 4-5^{\circ}, 5-6^{\circ}\right)$. The cortical surface area $\left(\mathrm{mm}^{2}\right)$ was summed across hemispheres within each subject and divided by the visual field area $\left(\mathrm{deg}^{2}\right)$.

Wedge ROls were computed in the following steps: First, area V1 and V2 were automatically labeled in the measured retinotopic maps of each hemisphere using a Bayesian inference algorithm [133]. Second, for each cardinal meridian and each $1^{\circ}$ eccentricity bin, we calculated the mean distance along the cortex to reach a $10^{\circ}$ polar angle. All vertices that fell within the eccentricity bin and polar angle distance were included in the particular ROI. We computed wedge strips, rather than an entire wedge or line to avoid localization errors in defining the exact boundaries. ROls used for the upper and lower vertical meridian included both V1 and V2 sections of the vertical meridian, assuming that V2 is approximately the same size as V1. However, these ROIs may be slightly less precise than the horizontal meridian ROI.

For each eccentricity bin and cardinal meridian, mean and standard error V1-V2 CMF were computed from bootstrapped data across subjects (1000 iterations). Mean data for each cardinal meridian were fit with a linear function in log-log space (i.e. power law function in linear coordinates) for $1-6^{\circ}$ eccentricity. 


\section{Convergence ratios}

The cone:mRGC ratio was computed by dividing $\mathrm{mRGC}$ density (cells/deg ${ }^{2}$ ) by cone density (cells $/ \mathrm{deg}^{2}$ ) for $0-40^{\circ}$ eccentricity, in $0.05^{\circ}$ bins. When density data were separated by cardinal meridian, data for nasal and temporal retina were averaged into one horizontal meridian. The $\mathrm{mRGC}: C M F$ ratio was computed in cells $/ \mathrm{mm}^{2}$. When comparing $\mathrm{mRGC}$ density to Horton \& Hoyt's CMF prediction, mRGC density (cells/deg ${ }^{2}$ ) was divided by V1 CMF $\left(\mathrm{deg}^{2} / \mathrm{mm}^{2}\right)$ for 0-40 eccentricity, in $0.05^{\circ}$ bins. When comparing HCP's retinotopy CMF to mRGC density, mRGC density was restricted to $1-6^{\circ}$ eccentricity, and divided by the power law functions fitted to the V1-V2 CMF.

\section{Asymmetry computation}

Radial asymmetries between meridians for cone density and mRGC density were calculated as percent change in retinal coordinates as in Equation 1 and 2:

Horizontal Vertical Asymmetry $=100 \cdot \frac{\text { mean (nasal,temporal })- \text { mean (superior,inferior })}{\text { mean(nasal,temporal,superior, } \text {,inferior })}$

Vertical Meridian Asymmetry $=100 \cdot \frac{\text { superior }- \text { inferior }}{\text { mean }(\text { superior }, \text { inferior })}$

Radial asymmetries in V1 CMF and behavior were computed with the same equations, but for visual field coordinates (i.e. nasal and temporal retina are left and right visual field meridians, and superior and inferior retina are lower and upper visual field meridians).

\section{Computational observer model}

The computational observer uses and extends a published model and simulated data [46]. The extensions include a midget RGC layer between the cone isomerization stage and the behavioral inference stage, and adding an SNR inference engine. The few occasions that additional cone absorption data needed to be simulated (e.g. higher stimulus contrasts for the SNR observer), we set the random number generator to the identical seed used previously to replicate the cone mosaic data. Given that the model and cone absorption data for simulated experiment are identical to those to the previous study, we refer to those methods on Scene radiance, Retinal irradiance, Cone mosaic and absorptions, Eye movements, and the Behavioral inference using a linear SVM classifier. 


\section{Stimulus parameters}

The computational model simulates a 2-AFC orientation discrimination task while varying stimulus contrast. The stimulus parameters are chosen to match the baseline condition of the psychophysical study by Himmelberg et al. [28], whose results have replicated the psychophysical study used for comparison in our previous computational observer model [11]. The recent psychophysics experiment used achromatic oriented Gabor patches, $\pm 15^{\circ}$ oriented from vertical, with a spatial frequency of 4 cycles per degree. Stimuli were presented at $4.5^{\circ}$ isoeccentric locations on the cardinal meridians, with a size of $3 \times 3^{\circ}$ visual angle $\left(\sigma=0.43^{\circ}\right)$ and duration of $120 \mathrm{~ms}$. These stimulus parameters were identical to those the model, except for size, duration and phase randomization of the Gabor. The simulated stimulus by the model was smaller ( $2 \times 2^{\circ}$ visual angle $\left(\sigma=0.25^{\circ}\right)$, shorter (54-ms, 2-ms sampling) without stimulus on- of offset period, and the phase of the Gabor patches was either $90^{\circ}$ or $270^{\circ}$, which was randomized across trials.

\section{Midget RGC layer}

Prior to the mRGC layer, Gabor stimuli were simulated as spectral scene radiance from a visual display, passed through the simulated human optics, subject to small fixational eye movements and isomerization by the cones in a rectangular mosaic ( $2 \times 2^{\circ}$ field-of-view) and saved as separate files for each stimulus contrast. The mRGC layer loaded the simulated 2D time-varying cone absorptions.

The mRGC layer was built as a rectangular array, with the identical size mosaic as the cone mosaic $\left(2 \times 2^{\circ}\right)$. Spatial summation by RGC RFs was implemented as 2D Difference of Gaussian (DoG) filters $[79,80]$. To define the size of the DoG RF, we first defined the mRGC:cone ratio parameter. We do not model on- and off-center mRGCs separately, assuming that one linear $m R G C$ (no rectification) represented a pair of rectified on- and off-centers. The variations in $\mathrm{mRGC}$ :cone ratios ranged from 2:1, 1:1, 0.67:1, 0.50:1, 0.40:1, where the highest ratio $(2: 1)$ is similar to the observed in the fovea and the lowest ratio $(0.4: 1)$ is similar to the observed at $\sim 12^{\circ}$ eccentricity [60]. These ratios spanned wide range because the purpose of the modeling was to assess how variation in $\mathrm{MRGC}$ spacing affects performance.

The DoG size was based on Croner \& Kaplan [81]: one standard deviation of the center Gaussian $\left(\sigma_{c}\right)$ was $1 / 3$ times the mRGC spacing. One standard deviation of the surround Gaussian $\left(\sigma_{s}\right)$ was 6 times the center standard deviation. The center/surround weights were 0.64:0.36, hence unbalanced. These parameters create neighboring DoG RFs that overlap at 
1.3 standard deviation from their centers, approximating RGC tiling in human retina based on overlap of dendrites fields [50].

The spatial computations of the mRGC layer were implemented in two stages. In the first stage, the 2D DoG filter was convolved with each 2D cone absorption frame separately for each time point and trial. The filtered cone responses were constrained such that the post-convolution array maintained the same size (using MATLAB's conv2 with shape variable set to 'same'). In the second stage, the filtered cone responses were linearly subsampled. This was implemented by resampling each row and column of the filtered cone responses with a sample rate equal to the $\mathrm{mRGC}$ :cone ratio. These spatially filtered and subsampled responses are the mRGC responses, which remained in units of photons/ms as there was no phototransduction from cone absorptions to cone current, and no spiking non-linearity implemented in this transformation. We did not implement Poisson noise in the $\mathrm{mRGC}$ layer.

\section{Simulated experiments}

A single simulated experiment had a total of 6,000 trials: 400 trials per contrast level, 200 clockwise and 200 counter-clockwise, which can be further subdivided in 100 trials with either a $90^{\circ}$ or $270^{\circ}$ stimulus phase. Stimulus contrast was systematically varied from 0 to $10 \%$ Michelson contrast, using 15 contrast levels ${ }^{1}$. The cone mosaic was identical across contrast levels, including the spatial distribution of L-, M-, and S- cones, the cone density, the cone spacing and the presence of fixational eye movements. Data from a single contrast level were represented as a 4D array ( $m$ rows by $n$ columns by 28 time points by 400 trials). The size of the $m$ by $n$ frame depended on the defined subsampling ratio used for the $\mathrm{mRGC}$ layer.

This single experiment was repeated for 13 different cone mosaics, which varied systematically in cone density and spacing. The cone density variation was implemented by simulating cone mosaics at different eccentricities, ranging from a density as high as at the fovea $\left(2.3 \times 10^{4}\right.$ cells $\left./ \mathrm{deg}^{2}\right)$ to as low as at $40^{\circ}$ eccentricity on the horizontal meridian $\left(0.047 \times 10^{4}\right.$ cells $\left./ \mathrm{deg}^{2}\right)$. This resulted in a total of 78,000 simulated trials $(6,000$ trials $\times 13$ cone densities).

Simulated experiments for each of the 13 different cone densities repeated for each of 5 different $\mathrm{mRGC}$ :cone ratios. Lastly, this entire simulation procedure was repeated 5 times, using

\footnotetext{
${ }^{1}$ Simulations for the cone mosaics with the three lowest densities and SNR observer required 9 additional contrasts levels ranging from $20 \%$ and $100 \%$ in order for the model performance to saturate at $100 \%$.
} 
a different random number generator seed for each replication iteration. This resulted in a total of $1,950,000$ simulated trials $(6,000$ trials $\times 13$ cone densities $\times 5$ ratios $\times 5$ replications).

\section{Inference engines}

The simulated trials were fed into an inference engine. The task of the inference engine was to classify if a trial contained a clockwise or counter-clockwise oriented Gabor stimulus given the $m R G C$ responses. Classification was performed separately for every 400 trials, i.e. separately for each contrast level, cone density, $\mathrm{mRGC}$ :cone ratio, and replication iteration of a single experiment. This classification routine was identical for each type of inference engine.

For the main analysis, we used a linear SVM classifier as implemented in MATLAB's fitcsvm with 10 -fold cross-validation and built-in z-scoring. This procedure is identical to the previously published model. Prior to classification, each 2D frame of $m R G C$ responses was transformed to the Fourier domain using a 2D FFT and the phase information was discarded. This transformation step was executed for each time point and trial separately. The mRGC amplitudes were concatenated across time and space, resulting in a single vector of trials by $\mathrm{mRGC}$ amplitudes. The order of the trials within this vector was randomized and fed into the linear SVM classifier with a set of stimulus labels. The classifier trained its weights on $90 \%$ of the trials, and tested on the $10 \%$ left-out trials. This resulted in accuracy (percent correct) for each given contrast level.

Accuracy data for a single simulated experiment were averaged across the five replication iterations. Error bars were computed for each contrast level as standard error across 1000 bootstraps, bootstrapping the mean across the 5 accuracy values with replacement. To extract the contrast threshold, a Weibull function was fitted to the average accuracy data. The threshold was defined as the power of 1 over the slope of the Weibull function, which comes out approximately $\sim 80 \%$ correct, given that chance is $50 \%$ for a 2 -AFC task and our slope was defined as $\beta=3$.

For the comparing inference engines in Fig 5, we used an ideal observer, a linear SVM computational observer, and an SNR computational observer. The implementation of the ideal and linear SVM model were identical to the previous published study [46]. Therefore, we only describe the details for the SNR computational observer below.

The SNR computational observer makes its decision based only on the signal-to-noise ratio and was implemented using MATLAB's snr function. From all three inference engines, this 
observer provides a lower bound on model performance as it has no knowledge about the stimulus or the task, and does not train on a set of trials to learn a stimulus template. Because the SNR observer has no knowledge about the stimulus or the task, it performs a same/different task rather than a classification task.

The SNR observer decides whether a stimulus is the same or different based on two inputs: one distribution of $\mathrm{mRGC}$ responses containing signal and one containing noise. The signal and noise distributions are defined by using a simplified version of the model as it has only one source of noise (photon noise), a cone mosaic with only one cone type (L-cones), no fixational eye movements, and no stimulus phase shifts. We define signal by taking the difference in noiseless clockwise and counter-clockwise mRGC responses for each 2-ms frame within a trial. The noise is defined as the background photon noise by subtracting the noiseless $m R G C$ responses from $m R G C$ responses with photon noise for each 2-ms frame within a trial. Here, we assume that the spread of the pooled noise distribution is the same for the two stimulus classes, thus we can define the noise from either stimulus classes (in this case we use clockwise trials). These signal and noise input distributions are a 3D-array with the dimensions time points $\mathrm{x}$ mRGCs $\mathrm{x}$ trials.

The signal and noise distributions were then vectorized by concatenating all responses of all trials and input of the snr function. This function first computed the magnitude in signal and noise by taking the sums of squares of each vector and are in units of decibel, and then divides the signal magnitude by the noise magnitude to get the signal-to-noise ratio in decibel. To compare the observer's performance to other inference engines, we converted the output from magnitude $(\mathrm{dB})$ to discriminability-index (d'), and convert d' to percent correct. Doing so, we assume that the SNR observer sets an unbiased criterion to determine whether the signal component comes from two of the same or different stimuli. By comparing the SNR observer to the ideal and SVM observer, we make a second assumption: performing a same/different task and performing a 2-AFC discrimination task result in similar performance.

\section{Comparing model performance to behavior}

To quantify the contribution of the spatial filtering by mRGCs, we compared the model performance to behavior reported by Himmelberg et al. [28]. First, we extracted the contrast thresholds across cone density and fitted a linear function in log-log space (i.e. power law function) to the contrast thresholds, separately for each simulated mRGC:cone ratio. 
Second, we placed these fits for each ratio in a 3D coordinate space: where the $\mathrm{x}$ dimension represents cone density, $y$-dimension represents the $\mathrm{mRGC}$ to cone ratio, and the $z$ dimension represents the predicted contrast threshold by the linear fits in log-log space. To get a finer representation of predicted contrast thresholds as a function of $\mathrm{mRGC}$ :cone ratio, we linearly interpolated thresholds along the mRGC:cone ratio axis by a factor of 8 . This resulted in $\mathrm{mRGC}$ :cone ratios at a sample rate of 0.1 . For visualization purposes of the 3D mesh, cone density was resampled to have equal log10 spaced values.

Third, we calculated the observed cone density and $\mathrm{mRGC}$ :cone ratio at $4.5^{\circ}$ eccentricity for the four cardinal meridians using data from Curcio et al. [7] and Watson [60]. With the linear interpolation, maximum error for the calculated ratio did not exceed \pm 0.014 . This calculation results in four [cone, $\mathrm{mRGC}$ ]-density coordinates to extract predicted contrast thresholds by our model for each cardinal meridian at $4.5^{\circ}$ eccentricity. Predicted thresholds for the 'model up to cones' were computed using previously published mean contrast thresholds [46] (varying cone density condition). These data were fitted with a linear function in log-log space to match the power function used for fitting the contrast thresholds for the mRGC model.

Contrast thresholds were converted into contrast sensitivity by taking the reciprocal. Nasal and temporal retina are averaged to represent the horizontal meridian. Because cone density can vary dramatically across observers [134, 135], we computed error bars that represent the amount of variability in predicted sensitivity based on a difference in underlying cone density (instead of caused by the random number generator seed as in Supplemental Fig 2 and 3).

The upper/lower bound of the error bars in cone and mRGC model predictions were defined by assuming that our estimates of cone density on the meridians are imperfect. Specifically, we assumed that the measured asymmetries might be off by as much as a factor of 2. So, for example, if the reported density for the horizontal meridian is $20 \%$ above the mean, and for the vertical meridian is $20 \%$ below the mean, we considered the possibility that they were in fact $40 \%$ above or below the mean, or $10 \%$ above or below the mean. 


\section{References}

1. Wertheim T. Über die indirekte Sehschärfe. Z Psychol, Physiol. 1894;7:172-83.

2. $\quad$ Ludvigh E. Extrafoveal acuity as measured with Snellen test-letters. American Journal of Ophtalmology. 1941;24:225-33.

3. Polyak SL. The retina. Press UoC, editor. Chicago1941.

4. Strasburger $\mathrm{H}$, Rentschler I, Juttner M. Peripheral vision and pattern recognition: a review. J Vis. 2011;11(5):13. doi: 10.1167/11.5.13. PubMed PMID: 22207654.

5. Hering E. Beiträge zur Physiologie [Contributions to physiology]. Leipzig: Wilhelm Engelmann; 1861.

6. von Helmholtz H. Handbuch der physiologischen Optik. Hamburg: Leopold Voss; 1896.

7. Curcio CA, Sloan KR, Kalina RE, Hendrickson AE. Human photoreceptor topography. J Comp Neurol. 1990;292(4):497-523. doi: 10.1002/cne.902920402. PubMed PMID: 2324310.

8. Song H, Chui TY, Zhong Z, Elsner AE, Burns SA. Variation of cone photoreceptor packing density with retinal eccentricity and age. Invest Ophthalmol Vis Sci. 2011;52(10):737684. doi: 10.1167/iovs.11-7199. PubMed PMID: 21724911; PubMed Central PMCID: PMCPMC3183974.

9. Mackeben M. Sustained focal attention and peripheral letter recognition. Spat Vis. 1999;12(1):51-72. Epub 1999/04/09. doi: 10.1163/156856899x00030. PubMed PMID: 10195388.

10. Carrasco M, Talgar CP, Cameron EL. Characterizing visual performance fields: effects of transient covert attention, spatial frequency, eccentricity, task and set size. Spat Vis. 2001;15(1):61-75. Epub 2002/03/15. PubMed PMID: 11893125; PubMed Central PMCID: PMCPMC4332623.

11. Cameron EL, Tai JC, Carrasco M. Covert attention affects the psychometric function of contrast sensitivity. Vision Res. 2002;42(8):949-67. Epub 2002/04/06. PubMed PMID: 11934448.

12. Corbett JE, Carrasco M. Visual performance fields: frames of reference. PLoS One. 2011;6(9):e24470. doi: 10.1371/journal.pone.0024470. PubMed PMID: 21931727; PubMed Central PMCID: PMCPMC3169603.

13. Talgar CP, Carrasco M. Vertical meridian asymmetry in spatial resolution: visual and attentional factors. Psychon Bull Rev. 2002;9(4):714-22. doi: 10.1093/mnras/94.5.377. PubMed PMID: 12613674.

14. Fuller S, Carrasco M. Perceptual consequences of visual performance fields: the case of the line motion illusion. J Vis. 2009;9(4):13 1-7. doi: 10.1167/9.4.13. PubMed PMID: 19757922; PubMed Central PMCID: PMCPMC3703960.

15. Rovamo J, Virsu V. An estimation and application of the human cortical magnification factor. Exp Brain Res. 1979;37(3):495-510. doi: 10.1007/BF00236819. PubMed PMID: 520439.

16. Virsu V, Rovamo J. Visual resolution, contrast sensitivity, and the cortical magnification factor. Exp Brain Res. 1979;37(3):475-94. PubMed PMID: 520438. 
17. Kroon JN, Rijsdijk JP, van der Wildt GJ. Peripheral contrast sensitivity for sine-wave gratings and single periods. Vision Res. 1980;20(3):243-52. doi: 10.1016/0042-6989(80)901091. PubMed PMID: 7385598.

18. Rijsdijk JP, Kroon JN, van der Wildt GJ. Contrast sensitivity as a function of position on the retina. Vision Res. 1980;20(3):235-41. doi: 10.1016/0042-6989(80)90108-X. PubMed PMID: 7385597.

19. Robson JG, Graham N. Probability summation and regional variation in contrast sensitivity across the visual field. Vision Res. 1981;21(3):409-18. doi: 10.1016/00426989(81)90169-3. PubMed PMID: 7269319.

20. Lundh BL, Lennerstrand G, Derefeldt G. Central and peripheral normal contrast sensitivity for static and dynamic sinusoidal gratings. Acta Ophthalmol (Copenh). 1983;61(2):171-82. Epub 1983/04/01. doi: 10.1111/j.1755-3768.1983.tb01410.x. PubMed PMID: 6880630 .

21. Skrandies W. Human contrast sensitivity: regional retinal differences. Hum Neurobiol. 1985;4(2):97-9. PubMed PMID: 4030428.

22. Seiple W, Holopigian K, Szlyk JP, Wu C. Multidimensional visual field maps: relationships among local psychophysical and local electrophysiological measures. J Rehabil Res Dev. 2004;41(3A):359-72. PubMed PMID: 15543452.

23. Silva MF, Maia-Lopes S, Mateus C, Guerreiro M, Sampaio J, Faria P, et al. Retinal and cortical patterns of spatial anisotropy in contrast sensitivity tasks. Vision Res. 2008;48(1):12735. doi: 10.1016/j.visres.2007.10.018. PubMed PMID: 18067943.

24. Silva MF, Mateus C, Reis A, Nunes S, Fonseca P, Castelo-Branco M. Asymmetry of visual sensory mechanisms: electrophysiological, structural, and psychophysical evidences. J Vis. 2010;10(6):26. doi: 10.1167/10.6.26. PubMed PMID: 20884575.

25. Abrams J, Nizam A, Carrasco M. Isoeccentric locations are not equivalent: the extent of the vertical meridian asymmetry. Vision Res. 2012;52(1):70-8. Epub 2011/11/17. doi:

10.1016/j.visres.2011.10.016. PubMed PMID: 22086075; PubMed Central PMCID: PMCPMC3345502.

26. Baldwin AS, Meese TS, Baker DH. The attenuation surface for contrast sensitivity has the form of a witch's hat within the central visual field. J Vis. 2012;12(11). Epub 2012/10/30. doi: 10.1167/12.11.23. PubMed PMID: 23104816.

27. Silva MF, d'Almeida OC, Oliveiros B, Mateus C, Castelo-Branco M. Development and aging of visual hemifield asymmetries in contrast sensitivity. J Vis. 2014;14(12). doi:

10.1167/14.12.19. PubMed PMID: 25326605.

28. Himmelberg MM, Winawer J, Carrasco M. Stimulus-dependent contrast sensitivity asymmetries around the visual field. Journal of Vision. 2020;In press. doi:

10.1101/2020.06.30.181156.

29. Altpeter E, Mackeben M, Trauzettel-Klosinski S. The importance of sustained attention for patients with maculopathies. Vision Res. 2000;40(10-12):1539-47. doi: 10.1016/S00426989(00)00059-6. PubMed PMID: 10788657.

30. Carrasco M, Williams PE, Yeshurun Y. Covert attention increases spatial resolution with or without masks: support for signal enhancement. J Vis. 2002;2(6):467-79. doi: 10.1167/2.6.4. PubMed PMID: 12678645. 
31. Montaser-Kouhsari L, Carrasco M. Perceptual asymmetries are preserved in short-term memory tasks. Atten Percept Psychophys. 2009;71(8):1782-92. doi: 10.3758/APP.71.8.1782. PubMed PMID: 19933562; PubMed Central PMCID: PMCPMC3697833.

32. Barbot A, Xue S, Carrasco M. Spatial frequency asymmetries around the visual field. PsyArXiv (Under review at Journal of Vision). 2020. doi: 10.31234/osf.io/ruwp9.

33. Fuller S, Rodriguez RZ, Carrasco M. Apparent contrast differs across the vertical meridian: visual and attentional factors. J Vis. 2008;8(1):16 1-. doi: 10.1167/8.1.16. PubMed PMID: 18318619; PubMed Central PMCID: PMCPMC2789458.

34. Chaikin JD, Corbin HH, Volkmann J. Mapping a field of short-time visual search. Science. 1962;138(3547):1327-8. doi: 10.1126/science.138.3547.1327. PubMed PMID: 14019856.

35. Krose BJ, Julesz B. The control and speed of shifts of attention. Vision Res. 1989;29(11):1607-19. PubMed PMID: 2635484.

36. Carrasco M, Giordano AM, McElree B. Temporal performance fields: visual and attentional factors. Vision Res. 2004;44(12):1351-65. doi: 10.1016/j.visres.2003.11.026. PubMed PMID: 15066395.

37. Rezec AA, Dobkins KR. Attentional weighting: a possible account of visual field asymmetries in visual search? Spat Vis. 2004;17(4-5):269-93. PubMed PMID: 15559106.

38. Pretorius LL, Hanekom JJ. An accurate method for determining the conspicuity area associated with visual targets. Hum Factors. 2006;48(4):774-84. doi:

10.1518/001872006779166370. PubMed PMID: 17240724.

39. Kristjansson A, Sigurdardottir HM. On the benefits of transient attention across the visual field. Perception. 2008;37(5):747-64. doi: 10.1068/p5922. PubMed PMID: 18605148.

40. Najemnik J, Geisler WS. Eye movement statistics in humans are consistent with an optimal search strategy. J Vis. 2008;8(3):4 1-14. doi: 10.1167/8.3.4. PubMed PMID: 18484810; PubMed Central PMCID: PMCPMC2868380.

41. Najemnik J, Geisler WS. Simple summation rule for optimal fixation selection in visual search. Vision Res. 2009;49(10):1286-94. doi: 10.1016/j.visres.2008.12.005. PubMed PMID: 19138697.

42. Fortenbaugh FC, Silver MA, Robertson LC. Individual differences in visual field shape modulate the effects of attention on the lower visual field advantage in crowding. $J$ Vis. 2015;15(2). doi: 10.1167/15.2.19. PubMed PMID: 25761337; PubMed Central PMCID: PMCPMC4327314.

43. Toet A, Levi DM. The two-dimensional shape of spatial interaction zones in the parafovea. Vision Res. 1992;32(7):1349-57. Epub 1992/07/01. doi: 10.1016/00426989(92)90227-a. PubMed PMID: 1455707.

44. He S, Cavanagh P, Intriligator J. Attentional resolution and the locus of visual awareness. Nature. 1996;383(6598):334-7. doi: 10.1038/383334a0. PubMed PMID: 8848045.

45. Greenwood JA, Szinte M, Sayim B, Cavanagh P. Variations in crowding, saccadic precision, and spatial localization reveal the shared topology of spatial vision. Proc Natl Acad Sci U S A. 2017;114(17):E3573-E82. Epub 2017/04/12. doi: 10.1073/pnas.1615504114. PubMed PMID: 28396415; PubMed Central PMCID: PMCPMC5410794. 
46. Kupers ER, Carrasco M, Winawer J. Modeling visual performance differences 'around' the visual field: A computational observer approach. PLoS Comput Biol. 2019;15(5):e1007063. Epub 2019/05/28. doi: 10.1371/journal.pcbi.1007063. PubMed PMID: 31125331; PubMed Central PMCID: PMCPMC6553792.

47. Drasdo N. Receptive field densities of the ganglion cells of the human retina. Vision Res. 1989;29(8):985-8. Epub 1989/01/01. doi: 10.1016/0042-6989(89)90113-2. PubMed PMID: 2629213.

48. Curcio CA, Allen KA. Topography of ganglion cells in human retina. J Comp Neurol. 1990;300(1):5-25. Epub 1990/10/01. doi: 10.1002/cne.903000103. PubMed PMID: 2229487.

49. Dacey DM, Petersen MR. Dendritic field size and morphology of midget and parasol ganglion cells of the human retina. Proc Natl Acad Sci U S A. 1992;89(20):9666-70. doi: 10.1073/pnas.89.20.9666. PubMed PMID: 1409680; PubMed Central PMCID: PMCPMC50193.

50. Dacey DM. The mosaic of midget ganglion cells in the human retina. J Neurosci. 1993;13(12):5334-55. PubMed PMID: 8254378.

51. Sjostrand J, Popovic Z, Conradi N, Marshall J. Morphometric study of the displacement of retinal ganglion cells subserving cones within the human fovea. Graefes Arch Clin Exp Ophthalmol. 1999;237(12):1014-23. Epub 2000/02/02. doi: 10.1007/s004170050338. PubMed PMID: 10654171.

52. Drasdo N, Millican CL, Katholi CR, Curcio CA. The length of Henle fibers in the human retina and a model of ganglion receptive field density in the visual field. Vision Res.

2007;47(22):2901-11. Epub 2007/02/27. doi: 10.1016/j.visres.2007.01.007. PubMed PMID: 17320143; PubMed Central PMCID: PMCPMC2077907.

53. Liu Z, Kurokawa K, Zhang F, Lee JJ, Miller DT. Imaging and quantifying ganglion cells and other transparent neurons in the living human retina. Proc Natl Acad Sci U S A. 2017;114(48):12803-8. Epub 2017/11/16. doi: 10.1073/pnas.1711734114. PubMed PMID: 29138314; PubMed Central PMCID: PMCPMC5715765.

54. Webb SV, Kaas JH. The sizes and distribution of ganglion cells in the retina of the owl monkey. Aotus trivirgatus. Vision Res. 1976;16(11):1247-54. Epub 1976/01/01. doi: 10.1016/0042-6989(76)90049-3. PubMed PMID: 827113.

55. Perry VH, Oehler R, Cowey A. Retinal ganglion cells that project to the dorsal lateral geniculate nucleus in the macaque monkey. Neuroscience. 1984;12(4):1101-23. PubMed PMID: 6483193.

56. Perry $\mathrm{VH}$, Cowey A. The ganglion cell and cone distributions in the monkey's retina: Implications for central magnification factors. Vision Res. 1985;25(12):1795-810.

57. Wassle H, Grunert U, Rohrenbeck J, Boycott BB. Cortical magnification factor and the ganglion cell density of the primate retina. Nature. 1989;341(6243):643-6. Epub 1989/10/19. doi: 10.1038/341643a0. PubMed PMID: 2797190.

58. Leventhal AG, Rodieck RW, Dreher B. Retinal ganglion cell classes in the Old World monkey: morphology and central projections. Science. 1981;213(4512):1139-42. Epub 1981/09/04. doi: 10.1126/science.7268423. PubMed PMID: 7268423.

59. Curcio CA, Sloan KR, Jr., Packer O, Hendrickson AE, Kalina RE. Distribution of cones in human and monkey retina: individual variability and radial asymmetry. Science.

1987;236(4801):579-82. doi: 10.1126/science.3576186. PubMed PMID: 3576186. 
60. Watson $A B$. A formula for human retinal ganglion cell receptive field density as a function of visual field location. J Vis. 2014;14(7). doi: 10.1167/14.7.15. PubMed PMID: 24982468.

61. Horton JC, Hoyt WF. The representation of the visual field in human striate cortex. A revision of the classic Holmes map. Arch Ophthalmol. 1991;109(6):816-24. PubMed PMID: 2043069.

62. Polyak S. The main afferent fiber systems of the cerebral cortex in primates. Berkeley: University of California; 1932.

63. Polyak S. A contribution of the cerebral representation of the retina. The Journal of Comparative Neurology. 1933;57:541-617.

64. Pointer JS. The cortical magnification factor and photopic vision. Biol Rev Camb Philos Soc. 1986;61(2):97-119. Epub 1986/05/01. doi: 10.1111/j.1469-185x.1986.tb00463.x. PubMed PMID: 3527286.

65. Tolhurst DJ, Ling L. Magnification factors and the organization of the human striate cortex. Hum Neurobiol. 1988;6(4):247-54. Epub 1988/01/01. PubMed PMID: 2832355.

66. Adams DL, Horton JC. A precise retinotopic map of primate striate cortex generated from the representation of angioscotomas. J Neurosci. 2003;23(9):3771-89. Epub 2003/05/09. PubMed PMID: 12736348.

67. Myerson J, Manis PB, Miezin FM, Allman JM. Magnification in striate cortex and retinal ganglion cell layer of owl monkey: a quantitative comparison. Science. 1977;198(4319):855-7. Epub 1977/11/25. doi: 10.1126/science.411172. PubMed PMID: 411172.

68. Van Essen DC, Newsome WT, Maunsell JH. The visual field representation in striate cortex of the macaque monkey: asymmetries, anisotropies, and individual variability. Vision Res. 1984;24(5):429-48. Epub 1984/01/01. doi: 10.1016/0042-6989(84)90041-5. PubMed PMID: 6740964.

69. Farrell JE, Winawer J, Brainard DH, Wandell B. 27.2: Distinguished Paper: Modeling visible differences: The computational observer model. SID Symposium Digest of Technical Papers2014. p. 352-6.

70. Brainard DH, Jiang H, Cottaris NP, Rieke F, Chichilnisky EJ, Farrell JE, et al. ISETBIO: Computational tools for modeling early human vision. Imaging and Applied Optics 2015; 2015/06/07; Arlington, Virginia: Optical Society of America; 2015.

71. Cottaris N, Jiang H, Ding X, Wandell B, Brainard DH. A computational observer model of spatial contrast sensitivity: Effects of wavefront-based optics, cone mosaic structure, and inference engine. bioRxiv. 2018.

72. Barnett M, Aguirre GK, editors. A spatial model of human retinal cell densities and solution for retinal ganglion cell displacement. Vision Sciences Society Annual Meeting; 2018; St. Pete Beach, FL, USA: Journal of Vision.

73. Fahle M, Schmid M. Naso-temporal asymmetry of visual perception and of the visual cortex. Vision Res. 1988;28(2):293-300. Epub 1988/01/01. doi: 10.1016/0042-6989(88)90157-5. PubMed PMID: 3414016.

74. Benson NC, Kupers ER, Barbot A, Carrasco M, Winawer J. Cortical magnification in human visual cortex parallels task performance around the visual field. BioRXiv. 2020. doi: 10.1101/2020.08.26.268383. 
75. Benson NC, Jamison KW, Arcaro MJ, Vu AT, Glasser MF, Coalson TS, et al. The Human Connectome Project 7 Tesla retinotopy dataset: Description and population receptive field analysis. J Vis. 2018;18(13):23. Epub 2018/12/29. doi: 10.1167/18.13.23. PubMed PMID: 30593068; PubMed Central PMCID: PMCPMC6314247.

76. Mergenthaler K, Engbert R. Modeling the control of fixational eye movements with neurophysiological delays. Phys Rev Lett. 2007;98(13):138104. doi:

10.1103/PhysRevLett.98.138104. PubMed PMID: 17501244.

77. Martinez-Conde S, Macknik SL, Hubel DH. The role of fixational eye movements in visual perception. Nat Rev Neurosci. 2004;5(3):229-40. doi: 10.1038/nrn1348. PubMed PMID: 14976522.

78. Martinez-Conde S, Macknik SL, Troncoso XG, Hubel DH. Microsaccades: a neurophysiological analysis. Trends Neurosci. 2009;32(9):463-75. doi: 10.1016/j.tins.2009.05.006. PubMed PMID: 19716186.

79. Rodieck RW. Quantitative analysis of cat retinal ganglion cell response to visual stimuli. Vision Res. 1965;5(11):583-601. Epub 1965/12/01. doi: 10.1016/0042-6989(65)90033-7. PubMed PMID: 5862581.

80. Enroth-Cugell C, Robson JG. The contrast sensitivity of retinal ganglion cells of the cat. J Physiol. 1966;187(3):517-52. Epub 1966/12/01. doi: 10.1113/jphysiol.1966.sp008107. PubMed PMID: 16783910; PubMed Central PMCID: PMCPMC1395960.

81. Croner LJ, Kaplan E. Receptive fields of $P$ and $M$ ganglion cells across the primate retina. Vision Res. 1995;35(1):7-24. doi: 10.1016/0042-6989(94)E0066-T. PubMed PMID: 7839612.

82. Geisler WS. Physical limits of acuity and hyperacuity. J Opt Soc Am A. 1984;1(7):77582. Epub 1984/07/01. PubMed PMID: 6747742.

83. Green DG. Regional variations in the visual acuity for interference fringes on the retina. J Physiol. 1970;207(2):351-6. Epub 1970/04/01. doi: 10.1113/jphysiol.1970.sp009065. PubMed PMID: 5499023; PubMed Central PMCID: PMCPMC1348710.

84. Williams DR. Aliasing in human foveal vision. Vision Res. 1985;25(2):195-205. Epub 1985/01/01. doi: 10.1016/0042-6989(85)90113-0. PubMed PMID: 4013088.

85. Coletta NJ, Williams DR. Psychophysical estimate of extrafoveal cone spacing. J Opt Soc Am A. 1987;4(8):1503-13. Epub 1987/08/01. PubMed PMID: 3625330.

86. Williams DR, Coletta NJ. Cone spacing and the visual resolution limit. J Opt Soc Am A. 1987;4(8):1514-23. Epub 1987/08/01. PubMed PMID: 3625331.

87. Anderson SJ, Hess RF. Post-receptoral undersampling in normal human peripheral vision. Vision Res. 1990;30(10):1507-15. Epub 1990/01/01. doi: 10.1016/0042-6989(90)90031-f. PubMed PMID: 2247960.

88. Anderson SJ, Mullen KT, Hess RF. Human peripheral spatial resolution for achromatic and chromatic stimuli: limits imposed by optical and retinal factors. J Physiol. 1991;442:47-64. Epub 1991/10/01. PubMed PMID: 1798037; PubMed Central PMCID: PMCPMC1179877.

89. Banks MS, Sekuler AB, Anderson SJ. Peripheral spatial vision: limits imposed by optics, photoreceptors, and receptor pooling. J Opt Soc Am A. 1991;8(11):1775-87. Epub 1991/11/01. PubMed PMID: 1744774. 
90. Sjostrand J, Olsson V, Popovic Z, Conradi N. Quantitative estimations of foveal and extra-foveal retinal circuitry in humans. Vision Res. 1999;39(18):2987-98. Epub 2000/02/09. doi: 10.1016/s0042-6989(99)00030-9. PubMed PMID: 10664798.

91. Popovic Z, Sjostrand J. Resolution, separation of retinal ganglion cells, and cortical magnification in humans. Vision Res. 2001;41(10-11):1313-9. Epub 2001/04/27. doi: 10.1016/s0042-6989(00)00290-x. PubMed PMID: 11322976.

92. Popovic Z, Sjostrand J. The relation between resolution measurements and numbers of retinal ganglion cells in the same human subjects. Vision Res. 2005;45(17):2331-8. Epub 2005/06/01. doi: 10.1016/j.visres.2005.02.013. PubMed PMID: 15924946.

93. Wilkinson MO, Anderson RS, Bradley A, Thibos LN. Neural bandwidth of veridical perception across the visual field. J Vis. 2016;16(2):1. Epub 2016/01/30. doi: 10.1167/16.2.1. PubMed PMID: 26824638; PubMed Central PMCID: PMCPMC5833322.

94. Anstis SM. Letter: A chart demonstrating variations in acuity with retinal position. Vision Res. 1974;14(7):589-92. Epub 1974/07/01. doi: 10.1016/0042-6989(74)90049-2. PubMed PMID: 4419807.

95. Duncan RO, Boynton GM. Cortical magnification within human primary visual cortex correlates with acuity thresholds. Neuron. 2003;38(4):659-71. PubMed PMID: 12765616.

96. Schwarzkopf DS, Song C, Rees G. The surface area of human V1 predicts the subjective experience of object size. Nature neuroscience. 2011;14(1):28-30. doi: 10.1038/nn.2706. PubMed PMID: 21131954; PubMed Central PMCID: PMC3012031.

97. Liu T, Heeger DJ, Carrasco M. Neural correlates of the visual vertical meridian asymmetry. J Vis. 2006;6(11):1294-306. Epub 2007/01/11. doi: 10.1167/6.11.12. PubMed PMID: 17209736; PubMed Central PMCID: PMCPMC1864963.

98. Benson NC, Butt OH, Datta R, Radoeva PD, Brainard DH, Aguirre GK. The retinotopic organization of striate cortex is well predicted by surface topology. Curr Biol. 2012;22(21):2081 5. doi: 10.1016/j.cub.2012.09.014. PubMed PMID: 23041195; PubMed Central PMCID: PMC3494819.

99. Silva MF, Brascamp JW, Ferreira S, Castelo-Branco M, Dumoulin SO, Harvey BM. Radial asymmetries in population receptive field size and cortical magnification factor in early visual cortex. Neuroimage. 2018;167:41-52. Epub 2017/11/21. doi: 10.1016/j.neuroimage.2017.11.021. PubMed PMID: 29155078.

100. Daniel PM, Whitteridge $D$. The representation of the visual field on the cerebral cortex in monkeys. J Physiol. 1961;159:203-21. Epub 1961/12/01. doi: 10.1113/jphysiol.1961.sp006803. PubMed PMID: 13883391; PubMed Central PMCID: PMCPMC1359500.

101. Rolls ET, Cowey A. Topography of the retina and striate cortex and its relationship to visual acuity in rhesus monkeys and squirrel monkeys. Exp Brain Res. 1970;10(3):298-310. Epub 1970/01/01. doi: 10.1007/BF00235053. PubMed PMID: 4986000.

102. Braccini C, Gambardella G, Sandini G, Tagliasco V. A model of the early stages of the human visual system: functional and topological transformations performed in the peripheral visual field. Biol Cybern. 1982;44(1):47-58. Epub 1982/01/01. doi: 10.1007/BF00353955. PubMed PMID: 7093369.

103. Schutt HH, Wichmann FA. An image-computable psychophysical spatial vision model. J Vis. 2017;17(12):12. Epub 2017/10/21. doi: 10.1167/17.12.12. PubMed PMID: 29053781. 
104. Bradley C, Abrams J, Geisler WS. Retina-V1 model of detectability across the visual field. J Vis. 2014;14(12). Epub 2014/10/23. doi: 10.1167/14.12.22. PubMed PMID: 25336179; PubMed Central PMCID: PMCPMC4204678.

105. Watson $A B$, Ahumada AJ. Letter identification and the neural image classifier. J Vis. 2015;15(2). Epub 2015/03/12. doi: 10.1167/15.2.15. PubMed PMID: 25761333.

106. Kwon M, Liu R. Linkage between retinal ganglion cell density and the nonuniform spatial integration across the visual field. Proc Natl Acad Sci U S A. 2019;116(9):3827-36. Epub 2019/02/10. doi: 10.1073/pnas.1817076116. PubMed PMID: 30737290; PubMed Central PMCID: PMCPMC6397585.

107. Perry VH, Cowey A. The lengths of the fibres of Henle in the retina of macaque monkeys: implications for vision. Neuroscience. 1988;25(1):225-36. Epub 1988/04/01. doi: 10.1016/0306-4522(88)90021-8. PubMed PMID: 3393279.

108. Tootell RB, Switkes E, Silverman MS, Hamilton SL. Functional anatomy of macaque striate cortex. II. Retinotopic organization. The Journal of neuroscience : the official journal of the Society for Neuroscience. 1988;8(5):1531-68. Epub 1988/05/01. PubMed PMID: 3367210; PubMed Central PMCID: PMCPMC6569212.

109. Andrews TJ, Halpern SD, Purves D. Correlated size variations in human visual cortex, lateral geniculate nucleus, and optic tract. The Journal of neuroscience : the official journal of the Society for Neuroscience. 1997;17(8):2859-68. PubMed PMID: 9092607.

110. Kastner S, Schneider KA, Wunderlich K. Beyond a relay nucleus: neuroimaging views on the human LGN. Progress in brain research. 2006;155:125-43. doi: 10.1016/S00796123(06)55008-3. PubMed PMID: 17027384.

111. Malpeli JG, Baker FH. The representation of the visual field in the lateral geniculate nucleus of Macaca mulatta. J Comp Neurol. 1975;161(4):569-94. Epub 1975/06/15. doi: 10.1002/cne.901610407. PubMed PMID: 1133232.

112. Connolly M, Van Essen D. The representation of the visual field in parvicellular and magnocellular layers of the lateral geniculate nucleus in the macaque monkey. J Comp Neurol. 1984;226(4):544-64. Epub 1984/07/10. doi: 10.1002/cne.902260408. PubMed PMID: 6747034.

113. Schein SJ, de Monasterio FM. Mapping of retinal and geniculate neurons onto striate cortex of macaque. The Journal of neuroscience : the official journal of the Society for Neuroscience. 1987;7(4):996-1009. Epub 1987/04/01. PubMed PMID: 3033167; PubMed Central PMCID: PMCPMC6568992.

114. Azzopardi $P$, Cowey A. Preferential representation of the fovea in the primary visual cortex. Nature. 1993;361(6414):719-21. Epub 1993/02/25. doi: 10.1038/361719a0. PubMed PMID: 7680108.

115. Malpeli JG, Lee D, Baker FH. Laminar and retinotopic organization of the macaque lateral geniculate nucleus: magnocellular and parvocellular magnification functions. J Comp Neurol. 1996;375(3):363-77. Epub 1996/11/18. doi: 10.1002/(SICI)10969861(19961118)375:3<363::AID-CNE2>3.0.CO;2-0. PubMed PMID: 8915836.

116. Shriki $O$, Kohn A, Shamir M. Fast coding of orientation in primary visual cortex. PLoS Comput Biol. 2012;8(6):e1002536. Epub 2012/06/22. doi: 10.1371/journal.pcbi.1002536.

PubMed PMID: 22719237; PubMed Central PMCID: PMCPMC3375217. 
117. Barlow HB. Single units and sensation: a neuron doctrine for perceptual psychology? Perception. 1972;1(4):371-94. Epub 1972/01/01. doi: 10.1068/p010371. PubMed PMID: 4377168.

118. Cottaris NP, Jiang H, Ding X, Wandell BA, Brainard DH. A computational-observer model of spatial contrast sensitivity: Effects of wave-front-based optics, cone-mosaic structure, and inference engine. J Vis. 2019;19(4):8. doi: 10.1167/19.4.8. PubMed PMID: 30943530.

119. Cottaris NP, Wandell BA, Rieke F, Brainard DH. A computational observer model of spatial contrast sensitivity: Effects of photocurrent encoding, fixational eye movements and inference engine. Journal of Vision. 2020;in press. doi: https://doi.org/10.1101/759811.

120. Endeman D, Kamermans M. Cones perform a non-linear transformation on natural stimuli. J Physiol. 2010;588(Pt 3):435-46. Epub 2009/12/17. doi: 10.1113/jphysiol.2009.179036. PubMed PMID: 20008463; PubMed Central PMCID: PMCPMC2825609.

121. Gollisch T, Meister M. Eye smarter than scientists believed: neural computations in circuits of the retina. Neuron. 2010;65(2):150-64. Epub 2010/02/16. doi: 10.1016/j.neuron.2009.12.009. PubMed PMID: 20152123; PubMed Central PMCID: PMCPMC3717333.

122. Schwartz G, Rieke F. Perspectives on: information and coding in mammalian sensory physiology: nonlinear spatial encoding by retinal ganglion cells: when $1+1$ not equal 2 . J Gen Physiol. 2011;138(3):283-90. Epub 2011/08/31. doi: 10.1085/jgp.201110629. PubMed PMID: 21875977 ; PubMed Central PMCID: PMCPMC3171084.

123. Shapley R, Perry VH. Cat and monkey retinal ganglion cells and their visual functional roles. Trends in Neurosciences. 1986;9:229-35.

124. Rauschecker AM, Bowen RF, Parvizi J, Wandell BA. Position sensitivity in the visual word form area. Proc Natl Acad Sci U S A. 2012;109(24):E1568-77. doi: 10.1073/pnas.1121304109. PubMed PMID: 22570498; PubMed Central PMCID: PMC3386120.

125. Gibaldi A, Banks MS, editors. Nasotemporal division of retina is well suited for disparities of natural scenes. Vision Sciences Society Annual Meeting; 2019; St. Pete Beach, FL: Journal of Vision.

126. Aghajari S, Vinke LN, Ling S. Population spatial frequency tuning in human early visual cortex. J Neurophysiol. 2020;123(2):773-85. Epub 2020/01/16. doi: 10.1152/jn.00291.2019. PubMed PMID: 31940228; PubMed Central PMCID: PMCPMC7052645.

127. Campbell FW, Green DG. Monocular versus binocular visual acuity. Nature. 1965;208(5006):191-2. Epub 1965/10/09. doi: 10.1038/208191a0. PubMed PMID: 5884255.

128. Hubel DH, Wiesel TN. Receptive fields, binocular interaction and functional architecture in the cat's visual cortex. J Physiol. 1962;160:106-54. PubMed PMID: 14449617; PubMed Central PMCID: PMCPMC1359523.

129. Banton T, Levi DM. Binocular summation in vernier acuity. J Opt Soc Am A. 1991;8(4):673-80. PubMed PMID: 2045969.

130. Dougherty K, Cox MA, Westerberg JA, Maier A. Binocular Modulation of Monocular V1 Neurons. Current biology : CB. 2019;29(3):381-91 e4. doi: 10.1016/j.cub.2018.12.004. PubMed PMID: 30661798; PubMed Central PMCID: PMCPMC6363852.

131. Ugurbil K, Xu J, Auerbach EJ, Moeller S, Vu AT, Duarte-Carvajalino JM, et al. Pushing spatial and temporal resolution for functional and diffusion MRI in the Human Connectome Project. Neuroimage. 2013;80:80-104. Epub 2013/05/25. doi: 
10.1016/j.neuroimage.2013.05.012. PubMed PMID: 23702417; PubMed Central PMCID: PMCPMC3740184.

132. Van Essen DC, Smith SM, Barch DM, Behrens TE, Yacoub E, Ugurbil K, et al. The WUMinn Human Connectome Project: an overview. Neuroimage. 2013;80:62-79. doi:

10.1016/j.neuroimage.2013.05.041. PubMed PMID: 23684880; PubMed Central PMCID: PMC3724347.

133. Benson NC, Winawer J. Bayesian analysis of retinotopic maps. Elife. 2018;7. Epub 2018/12/07. doi: 10.7554/eLife.40224. PubMed PMID: 30520736; PubMed Central PMCID: PMCPMC6340702.

134. Roorda A, Williams DR. The arrangement of the three cone classes in the living human eye. Nature. 1999;397(6719):520-2. Epub 1999/02/24. doi: 10.1038/17383. PubMed PMID: 10028967.

135. Hofer H, Carroll J, Neitz J, Neitz M, Williams DR. Organization of the human trichromatic cone mosaic. The Journal of neuroscience : the official journal of the Society for Neuroscience. 2005;25(42):9669-79. Epub 2005/10/21. doi: 10.1523/JNEUROSCI.2414-05.2005. PubMed PMID: 16237171. 


\section{Supplementary Material}
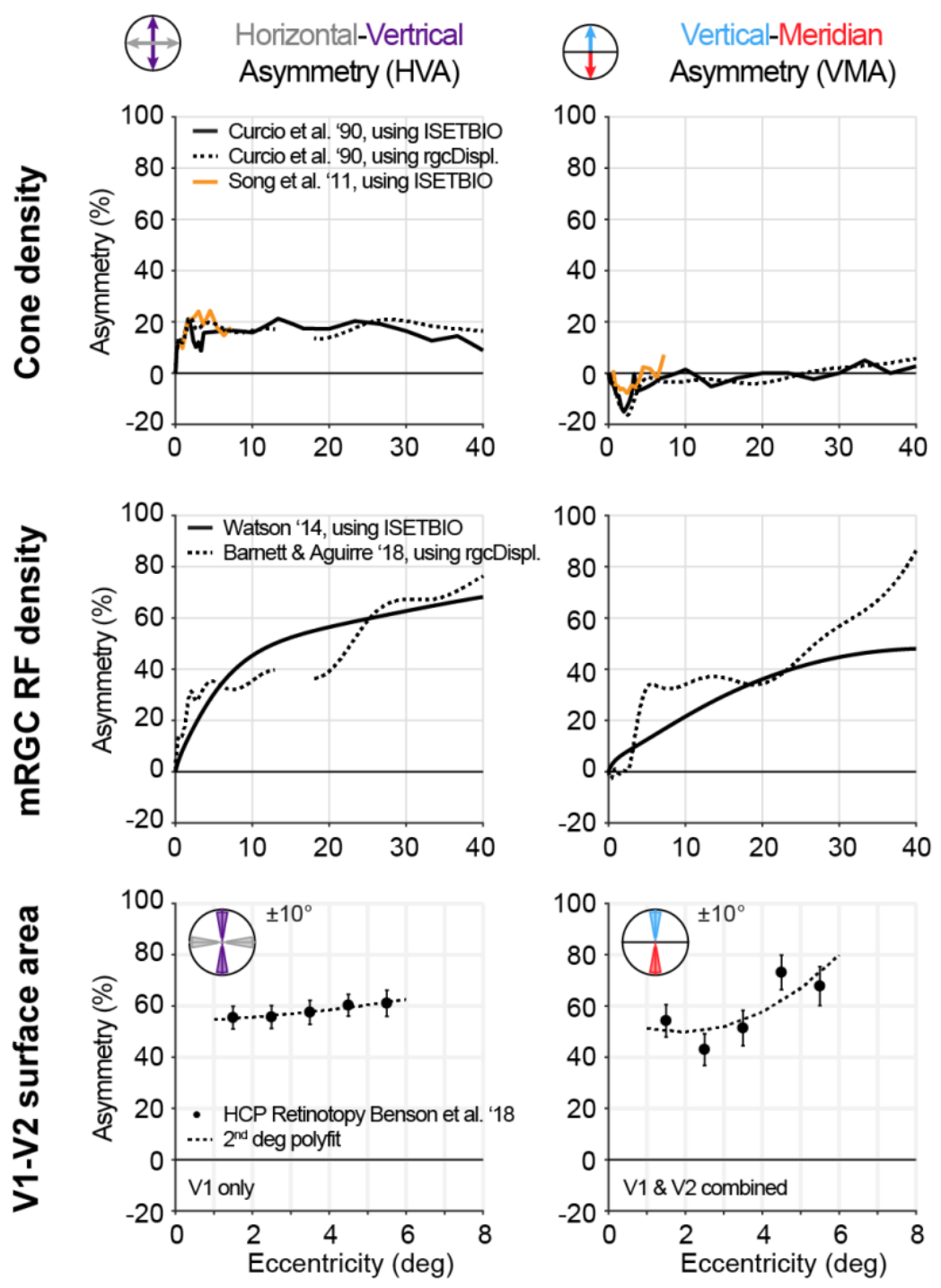

Supplemental Fig 1. Radial asymmetries for cone density, mRGC density and V1-V2 surface area computed from different publicly available datasets. Asymmetries are in percent change, calculated as the difference between horizontal and vertical meridians divided by their mean (left column), the difference between upper and lower vertical meridians divided by their means (right column). Positive asymmetries would positively correlate with observed differences in behavior. (Top row) Cone data are from either Curcio et al. [7] (black lines) or Song et al. [8] (orange line) computed with either ISETBIO (solid lines) or rgcDisplacementMap toolbox (dotted lines). (Middle row) Midget RGC RF data are computed using the computational model by Watson (2014) implemented in the ISETBio toolbox (solid black line) or Barnett \& Aguirre [72] implemented in the rgcDisplacementMap toolbox (dotted black line). (Bottom row) V1-V2 surface is computed from the Human Connectome Project 7T retinotopy dataset $(n=181)$, using the analyzed dataset by Benson et al. [74, 75]. Surface areas are defined as $\pm 10^{\circ}$ wedge ROls from $1-6^{\circ}$ eccentricity around the meridians, avoiding the central one degree and stimulus border $\left(7-8^{\circ}\right)$ as those data can be noisy. Note that the $x$-axis is truncated as cortical measurements are limited by the field-of-view in the fMRI experiment. Data are fit with a $2^{\text {nd }}$ degree polynomial, $R^{2}=0.54$ for horizontal-vertical and $R^{2}=0.81$ for verticalmeridian asymmetries). We use wedge ROls instead of line ROls because wedges are more robust to errors. For the horizontal meridian, wedge ROIs fall solely in V1, but for the vertical meridian wedges they fall partially on the V1-V2 dorsal and ventral borders. Here, we assume that area V2 is approximately the same size as V1, allowing us to estimate V1 vertical meridian surface area from V2. 
bioRxiv preprint doi: https://doi.org/10.1101/2020.10.20.347492; this version posted October 21, 2020. The copyright holder for this preprint (which was not certified by peer review) is the author/funder, who has granted bioRxiv a license to display the preprint in perpetuity. It is made available under aCC-BY-NC-ND 4.0 International license.
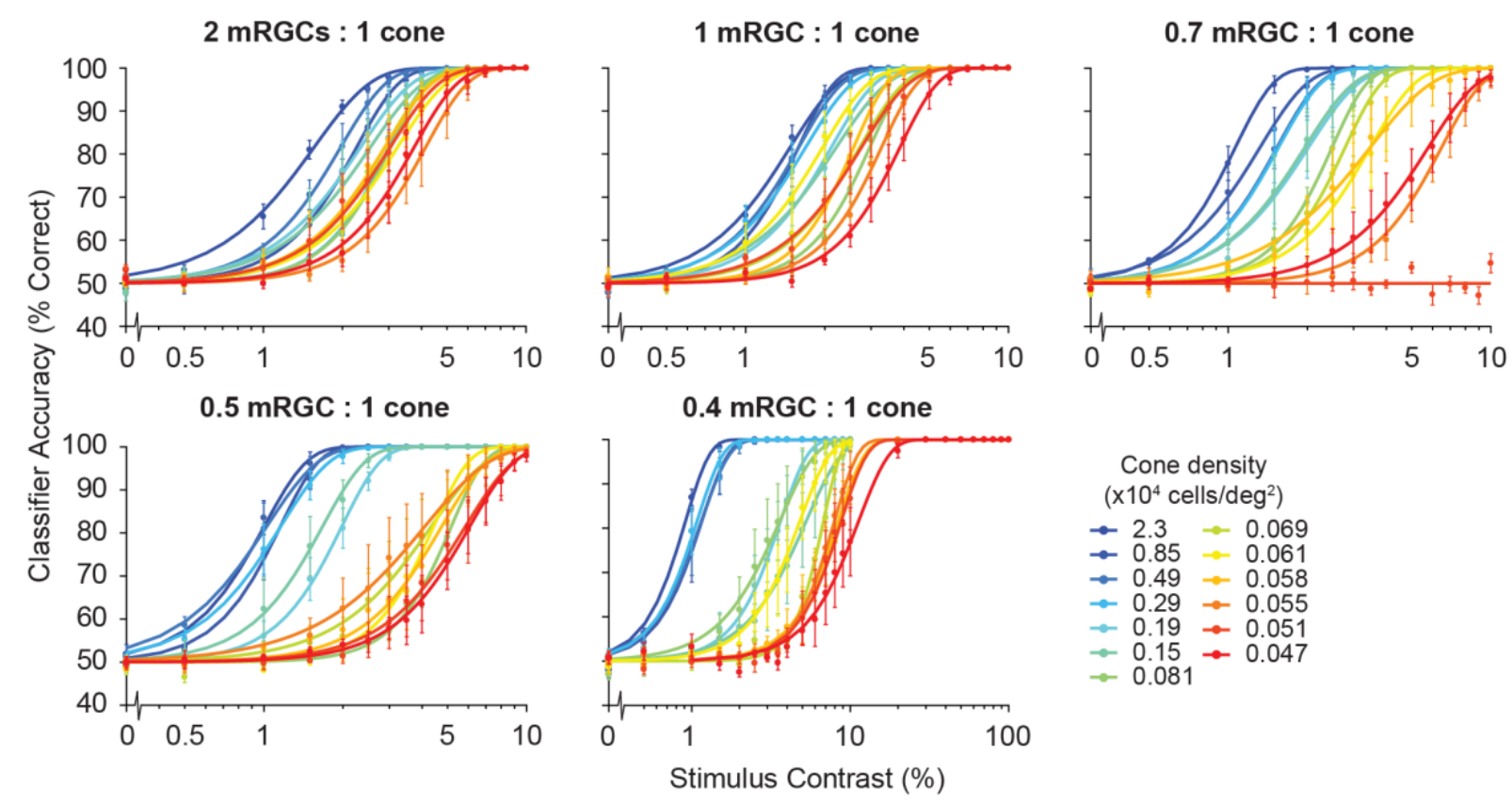

Cone density
$\left(\times 10^{4}\right.$ cells $/$ deg $\left.^{2}\right)$

$\rightarrow 2.3 \rightarrow 0.069$

$\rightarrow 0.85 \rightarrow 0.061$

$\rightarrow 0.49 \rightarrow 0.058$

$\rightarrow 0.29 \rightarrow 0.055$

$\rightarrow 0.19 \rightarrow 0.051$

$\rightarrow 0.15 \rightarrow 0.047$

Supplemental Fig 2. Classifier performance varying with cone density, separately for each mRGC:cone ratio. Classifier accuracy is averaged across 5 iterations of a simulated experiment with 200 clockwise and 200 counterclockwise trials. Average accuracy data are fitted with a Weibull function. Error bars represent standard error across 5 repeated experiments. The 0.4:1 mRGC:cone ratio (second row, middle column) is computed with higher contrasts for the cone mosaics with the three lowest cone densities (red to orange lines), as model performance did not saturate before $10 \%$ stimulus contrast.

A

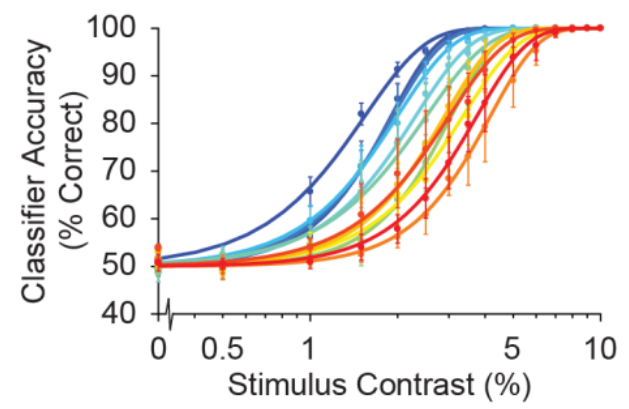

B

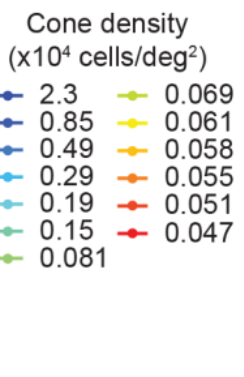

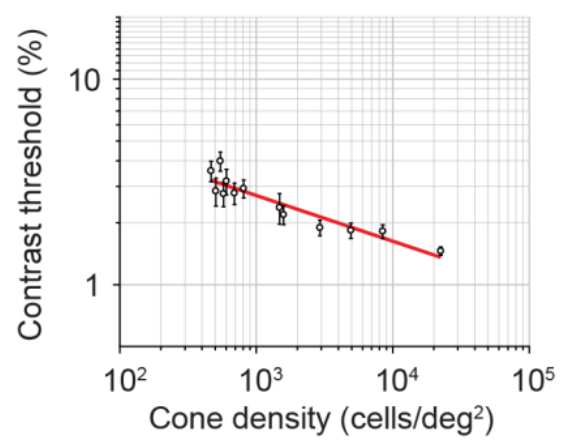

Supplemental Fig 3. Classifier performance for computational observer model up to cone isomerization. (A) Psychometric functions. Classifier accuracy is averaged across 5 iterations of a simulated experiment with 200 clockwise and 200 counter-clockwise trials. Average accuracy data are fitted with a Weibull function. (B) Contrast thresholds as a function of cone density. Data points are fitted with a linear function in log-log space (power law fit, red line). Data are averaged across 5 iterations of a simulated experiment varying in cone density. Error bars represent the standard error from bootstrapping the mean across 5 iterations, 1000 times with replacement. Goodness of fit for power law are $R^{2}=0.86$. 